\title{
Design of experiments for microbiological models
}

\author{
Holger Dette \\ Ruhr-Universität Bochum \\ Fakultät für Mathematik \\ 44780 Bochum \\ Germany
}

email: holger.dette@ruhr-uni-bochum.de

\author{
Viatcheslav B. Melas \\ St. Petersburg State University \\ Department of Mathematics \\ St. Petersburg \\ Russia \\ email: v.melas@pobox.spbu.ru
}

FAX: +492347094559

\author{
Nikolay Strigul \\ Princeton University, \\ Department of Ecology and \\ Evolutionary Biology \\ Princeton, NJ, USA \\ email: nstrigul@princeton.edu
}

\section{Introduction}

Methods of optimal experimental design potentially are very useful tool in research practice. There exists, unfortunately, some distance between mathematicians who investigating models of biological processes and practical researchers who do experiments. The beautiful, efficient and potentially very useful mathematical results are often not available for the wide range of experimental researchers which each time face the same problems. The purpose of this paper is to review applications of optimal experimental design in microbiology and to introduce those methods in such a way as to be accessible for scientists who making experimental research with no deep mathematical background. Examples in this paper are taken from microbiology but it should be also interesting for the wide group of researchers in biomedical sciences, such as biophysicists, pharmacologists, plant physiologists or for anyone facing the problem of identifying parameters of non-linear biological models.

The mathematical methods of optimal experimental design for non-linear models have been developed very intensively since $1960^{\text {th }}$ when the basic theorems by Kiefer and Wolfowitz were published 
(Kiefer, Wolfowitz, 1960; Kiefer, 1974). Then for several decades were very active theoretical research in this area and several reviews and monographs appeared (Fedorov, 1972; Pukelsheim, 1993; Walter, Pronzato, 1990; 1997). Now with the expansion of personal computers and the developing of efficient mathematical program packages such as Mathematica or Matlab and even more specialized software for the optimal experimental design searching (Rovati, 1988; Verotta, 1988, 1990; Marco et al., 2003) the application of these methods is becoming much more available for non-mathematicians and the frequency of application of this technique has significantly increased in the last 10 years.

Mathematical modeling is the usual method for describing quantitative data and predicting outcomes of microbiological processes (Pirt, 1975; Baranyi, Roberts, 1995). Several types of empirical regression models are used in applications. Most of the models, which describe microbial growth are non-linear first order differential equations in several variables. As a consequence the commonly used models in microbiology are regression models, where the unknown parameters enter the model nonlinearly. In the statistical literature these models are called nonlinear regression models. An appropriate choice of the experimental conditions can improve the quality of the experiment substantially. The present chapter is concerned with the optimal design of experiments for estimating parameters in nonlinear regression models. This problem has received much attention in the literature and the available references can be divided into the following three groups

(i) papers containing theoretical results on optimal experimental designs;

(ii) papers devoted to numerical construction of optimal designs or/and to stochastic modeling of biological data;

(iii) papers devoted to the application of optimal designs in real experiments.

Note that some of the references cited in this paper will fit in both groups (i) and (ii). However most papers can be put in group (ii) and only a few of the cited references belong to group (iii). The reason for the scarcity of papers in group (iii) may be that practitioners are not well-enough aware of the basic methods and advantages of optimal design.

The main purpose of this review is to explain some of the basic ideas of design of experiments and its applications in a form accessible to readers with a microbiological education. For this purpose we will avoid many technical details, which would require a deep mathematical background. Although nonlinear regression models appear in many applications, such as econometrics, genetics, agriculture etc., we will mainly concentrate on nonlinear models used in microbiology and will give a review of results from the literature on this issue.

The paper is organized in the following way. In Section 2 we will introduce some basic concepts and results relating to estimation and experimental designs for nonlinear regression models, used in microbiology. We discuss the properties of the least squares estimates, design optimality criteria and basic approaches to optimal design for nonlinear models. In Section 3 we discuss these 
concepts in more detail for the model given by the Monod differential equation. This model is taken as a typical example from microbiology to explain the practical benefits and difficulties in the implementation of optimal designs. The same section contains also a review of optimal designs for other typical nonlinear regression models in microbiology. A Bayesian approach to the construction of optimal designs is considered separately in Section 5, while Section 6 is devoted to some conclusions.

\section{Experimental design for nonlinear models}

In microbiological problems the quantity to be measured is usually dependent on one scalar control variable and a few parameters. The control variable is either the time of observation or a concentration of a substrate. For the sake of universality we will denote the control variable by the symbol $t$, inspite of the fact that in some microbiological applications it should be replaced by $c$, where $c$ is a concentration value. In many cases the variable $t$ is also called explanatory variable and due to natural biological conditions it is reasonable to assume that $t$ varies in an interval, say $[0, T]$, where $T$ is a maximal value for $t$. From a mathematical point of view observations taken under particular experimental conditions are random values. In microbiological applications these values can usually be considered as independent random variables. Supposing that our model describes a microbiological phenomenon adequately we can assume that each observation consists of a deterministic part, which describes the microbiological phenomenon, and a random error, which is modelled by a random variable with expectation equal to 0 . However, the variance of a particular observation (or of the corresponding error) can depend on the experimental condition $t$ under which the experiments have been performed. Generally speaking this dependence is unknown but we can hypothesize a mathematical model for it and then study the influence of the assumed model on the procedures for choosing experimental design and estimation of parameters. Thus we will assume that the experimental results at experimental conditions $t_{i}, i=1,2, \ldots, N$ (where $N$ is the total number of observations) are described by the following nonlinear regression model

$$
y_{i}=\eta\left(t_{j}, \theta\right)+g\left(t_{j}, \theta, \gamma\right) \varepsilon_{j}, j=1, \ldots, N
$$

where $y_{1}, \ldots, y_{N}$, are experimental observations taken under the experimental conditions $t_{1}, \ldots, t_{N}$. $\eta$ and $g$ are known functions, describing the microbiological phenomenon in the absence of observation errors, but the parameters $\theta=\left(\theta_{0}, \ldots, \theta_{m}\right)^{T}$ and $\gamma=\left(\gamma_{1}, \ldots, \gamma_{r}\right)^{T}$ are unknown parameters and have to be estimated from the available data $y_{1}, \ldots, y_{N}$. In many cases the main interest of the experimenter lies in a precise estimation of the parameters $\theta_{0}, \ldots, \theta_{m}$ while the parameters $\gamma_{1}, \ldots, \gamma_{r}$ can be considered as nuisance parameters [see Silvey (1980)]. Finally the random errors $\varepsilon_{1}, \ldots, \varepsilon_{N}$ are assumed to be independent and identically distributed random values with zero mean and variance 1 . For the sake of a transparent notation we will explain the main ideas of parameter estimation and design of experiments for nonlinear regression models under the as- 
sumption that the function $g$ is constant, say $g \equiv \sigma>0$, which corresponds to the case of a heteroscedastic nonlinear regression model. The main concepts in the heteroscedastic case, where the function $g$ is not constant, are exactly the same with an additional amount of notation [see e.g. Akinson and Cook (1995)].

Generally speaking, in microbiological problems the function $\eta(t, \theta)$ is given implicitly as a solution of an ordinary differential equation of the form

$$
\eta^{\prime}(t, \theta)=F(t, \theta, \eta(t, \theta))
$$

with an initial condition $\eta(0, \theta)=\theta_{0}$, where $\theta=\left(\theta_{0}, \ldots, \theta_{m}\right)^{T}$ and the function $F$ is explicitly given. Moreover, the function $F(t, \theta, \eta)$ is usually assumed to be representable in the form

$$
F(t, \theta, \eta)=\psi\left(t, \theta_{(1)}\right) \varphi\left(\eta, \theta_{(2)}\right), \eta=\eta(t, \theta)
$$

where $\left(\theta_{(1)}^{T}, \theta_{(2)}^{T}\right)=\theta^{T}$ and for fixed $\theta$ the function $\psi$ depends only on $t$, whereas the function $\varphi$ depends only on $\eta$. A few examples will be given soon in this section and some more examples are considered in section 3 .

Using (3), since $\eta^{\prime}(t, \theta)=d \eta / d t$, we obtain from (2) by multiplication of both sides by $d t$ and dividing then by $\varphi$ the following equation

$$
\left[1 / \varphi\left(\eta, \theta_{(2)}\right)\right] d \eta=\psi\left(t, \theta_{(1)}\right) d t
$$

Integrating both sides we obtain

$$
\Phi(\eta)=\Psi(t)
$$

where

$$
\begin{gathered}
\Phi(\eta):=\Phi\left(\eta, \theta_{(1)}\right)=\int_{0}^{\eta}\left[1 / \varphi\left(\eta, \theta_{(2)}\right)\right] d \eta, \eta=\eta(t, \theta), \\
\Psi(t):=\Psi\left(t, \theta_{(1)}\right)=\int_{0}^{t} \Psi\left(t, \theta_{(1)}\right) d \eta .
\end{gathered}
$$

Equation (4) determines the function $\eta=\eta(t, \theta)$ implicitly and also allows an efficient calculation of its values for any fixed value of $t$ and $\theta$. In a few important cases the function $\eta$ can be found explicitly. However, in most cases the function $\eta$ is only be defined implicitly.

\section{Example 2.1 The exponential regression model}

Let $\psi\left(t, \theta_{(1)}\right) \equiv 1, \varphi\left(\eta, \theta_{(2)}\right)=-\theta_{1} \eta$, then $\theta_{(2)}=\theta_{1}, \theta=\left(\theta_{0}, \theta_{1}\right)^{T}$ (note that $\theta_{(1)}$ is not needed here). It can be easily verified that

$$
\eta(t, \theta)=\theta_{0} e^{-\theta_{1} t}
$$

is the unique solution of equation (4). 


\section{Example 2.2 Three parameters logistic distribution}

Assume that $\psi\left(t, \theta_{(1)}\right) \equiv 1, \varphi\left(\eta, \theta_{(2)}=\theta_{1} \eta\left(1-\eta / \theta_{2}\right), \theta_{2}>0\right.$. Then we have

$$
\eta(t, \theta)=\frac{\theta_{0} e^{\theta_{2} t+\theta_{1}}}{1+e^{-\theta_{2} t+\theta_{1}}} .
$$

This function is called the three parameter logistic distribution.

\section{Example 2.3 The Monod differential equation}

Consider the equation (Monod, 1949; Pirt, 1975)

$$
\eta^{\prime}(t, \theta)=\mu(t) \eta(t, \theta)
$$

where $\theta=\left(\theta_{0}, \theta_{1}, \theta_{2}, \theta_{3}\right)^{T}$ denotes the unknown parameter

$$
\begin{aligned}
\mu(t) & =\mu(t, \theta)=\theta_{1} \frac{s(t)}{s(t)+\theta_{2}}, \\
s(t)-s_{0} & =\frac{\eta_{0}-\eta(t)}{\theta_{3}},
\end{aligned}
$$

and $s_{0}=s(\theta), \eta_{0}=\eta(\theta)=\theta_{0}$ are given initial condition and $\theta=\left(\theta_{1}, \theta_{2}, \theta_{3}\right)^{T}$. This model can be rewritten in the form

$$
\eta^{\prime}(t, \theta)=\varphi(\eta(t, \theta), \theta)
$$

where $\eta=\eta(t, \theta)$,

$$
\varphi(\eta, \theta)=\theta_{1} \frac{s_{0} \theta_{3}+\eta_{0}-\eta}{s_{0} \theta_{3}+\eta_{0}-\eta+\theta_{2} \theta_{3}} \eta
$$

By an integration we obtain equation (4) in the form

$$
t=\frac{1}{\theta_{1}}\left[(1+b) \ln \left(\eta / \eta_{0}\right)-b \ln \frac{c-\eta}{c-\eta_{0}}\right]
$$

where the constant $b$ is defined by $b=\theta_{2} \theta_{3} /\left[s_{0} \theta_{3}+\eta_{0}\right]$.

Assume that under the experimental condition $t_{j}(j=1,2, \ldots, n)$ an experiment is repeated $r_{j}$ times, while errors of different experiments under the same conditions are independent and let $\sum_{j=1}^{n} r_{j}=N$ denote the total number of observations in the experiment. The specification of the experimental conditions $t_{1}, \ldots, t_{n}$ and the relative proportions $\omega_{n}=\frac{r_{n}}{N}, \ldots, \omega_{1}=\frac{r_{1}}{N}$ of total observations taken under these conditions is called experimental design and denoted by a matrix

$$
\xi=\left(\begin{array}{c}
t_{1}, \ldots, t_{n} \\
\omega_{1}, \ldots, \omega_{n}
\end{array}\right)
$$


where $\sum_{j=1}^{n} \omega_{j}=1, \omega_{j}>0, t_{j} \in[0, T], j=1,2, \ldots, n$. In other words: a design of the form (5) advices the experimenter to take at each point $t_{j}$ exactly $r_{j}=\omega_{j} N$ observations $y_{i j}$ (if the numbers $\omega_{j} N$ are not integers a rounding procedure should be applied such that the resulting values $r_{j}$ satisfy $\left.\sum r_{j}=N\right)$. The results of the experiments from such type of design can be conveniently written in the form

$$
y_{j i}=\eta\left(t_{j}, \theta\right)+\varepsilon_{j i}, i=1, \ldots, r_{j}, j=1, \ldots, n,
$$

where $\varepsilon_{j i}$ denote independent random variables with expectation 0 and constant variance. Note that the index $j$ in (6) corresponds to the different experimental conditions $t_{j i}$ under which observations are obtained, while the index $i$ corresponds to the $r_{j}$ observations obtained under a particular fixed condition.

The most popular technique for estimating parameters is the least squares technique. The estimate $\hat{\theta}_{(N)}$ is obtained as the value $\theta$ for which the sum of squares

$$
\sum_{j=1}^{n} \sum_{i=1}^{r_{j}}\left(y_{i j}-\eta\left(t_{j}, \theta\right)\right)^{2} .
$$

attains its minimal value. Note that there may exist several values for $\theta$ minimizing this sum and this property depends on the particular regression function $\eta$ under consideration. As a consequence there may exist nonlinear regression models where the least squares estimator is not uniquely determined. However, in many models used in microbiology it can be proved by mathematical arguments that the least squares estimator is uniquely determined. For example, it was proved for the Monod model (Dette et. al., 2003) that for every $\theta^{0}$ with positive coordinates the least squares estimate is uniquely determined (note that in the Monod model the regression function can not be represented in an explicit form). The technique of that paper can be applied to most of the models used in microbiology.

Throughout this paper let $\theta^{*}$ denote the 'true' but unknown value of the parameter $\theta$. In other words the equation (6) holds for $\theta=\theta^{*}$. We assume that the least squares estimate $\hat{\theta}_{(N)}$ obtained from the $r_{j}$ experiments under the experimental conditions $t_{j}, j=1, \ldots, n$ is unique (note that $\sum_{j=1}^{n} r_{j}=N$ is the total number of observations). Under some assumptions of regularity it was shown by Jennrich (1969) that for a sufficiently large sample size $N$ the vector $\left(\theta_{(N)}-\theta^{*}\right)$ has approximately a normal distribution with zero mean and covariance matrix

$$
\frac{\sigma^{2}}{N} M^{-1}\left(\xi, \theta^{*}\right)
$$

where $\sigma$ denotes the standard deviation of the errors in model (6) and the matrix $M\left(\xi, \theta^{*}\right)$ is defined by

$$
M(\xi, \theta)=\left(\sum_{k=1}^{n} \omega_{k} \frac{\partial \eta\left(t_{k}, \theta\right)}{\partial \theta_{i}} \frac{\partial \eta\left(t_{k}, \theta\right)}{\partial \theta_{j}}\right)_{i, j=0}^{m}
$$

This matrix is called Fisher information matrix in the literature and we assume that it is nondegenerate throughout this paper. In principle the covariance matrix is a measure for the precision of 
the least squares estimator for the unknown parameter $\theta^{*}$ and a 'smaller' matrix yields more precise estimates. For example, the $i$ th diagonal element of the matrix $\frac{\sigma^{2}}{N} M^{-1}\left(\xi, \theta^{*}\right)$ will be denoted by $\left(\frac{\sigma^{2}}{N} M^{-1}\left(\xi, \theta^{*}\right)\right)_{i i}$ and is an approximation of the variance or mean squared error for the $i$ th component $\hat{\theta}_{i(N)}$ of the least squares estimator $\hat{\theta}_{(N)}$. A confidence interval for the $i$ th component $\theta_{i}$ of the vector $\theta$ is given by

$$
\left[\hat{\theta}_{i(N)}-\frac{\sigma u_{1-\frac{\alpha}{2}}}{\sqrt{N}} \sqrt{\left(M^{-1}\left(\xi, \theta^{*}\right)\right)_{i i}}, \hat{\theta}_{i(N)}+\frac{\sigma u_{1-\frac{\alpha}{2}}}{\sqrt{N}} \sqrt{\left(M^{-1}\left(\xi, \theta^{*}\right)\right)_{i i}}\right]
$$

where $u_{1-\alpha / 2}$ denotes the $1-\alpha / 2$ quantile of the standard normal distribution. If the sample size $N$ is sufficiently large this interval has a coverage probability approximately equal to $1-\alpha$. For the practical implementation of these results we will use the estimate $\hat{\theta}_{(N)}$ instead of the true vector $\theta^{*}$ since the last is unknown. In particular if we are interested in estimating the $i$-th parameter $\theta_{i}, i=0,1, \ldots, m$ we have the relation

$$
\mathcal{D}\left(\hat{\theta}_{i(N)}\right) \approx \frac{\sigma^{2}}{N}\left(M^{-1}\left(\xi, \hat{\theta}_{(N)}\right)\right)_{i i}
$$

and the confidence interval for the $i$ th component of the vector $\theta$ is given by

$$
\left[\hat{\theta}_{i(N)}-\frac{\hat{\sigma} u_{1-\frac{\alpha}{2}}}{\sqrt{N}} \sqrt{\left(\hat{M}^{-1}\left(\xi, \hat{\theta}_{(N)}\right)_{i i}\right.}, \hat{\theta}_{i(N)}+\frac{\hat{\sigma} u_{1-\frac{\alpha}{2}}}{\sqrt{N}} \sqrt{\left(M^{-1}\left(\xi, \hat{\theta}_{(N)}\right)_{i i}\right.}\right]
$$

where $\hat{\sigma}^{2}$ is an estimate of the unknown variance of the error. For a concrete nonlinear regression model closeness of the estimator $\hat{\theta}_{(N)}$ to $\theta^{*}$ and the variance $\mathcal{D}\left(\hat{\theta}_{i(N)}\right)$ to the variance $\frac{\sigma^{2}}{N}$ $\left(M^{-1}\left(\xi, \hat{\theta}_{(N)}\right)\right)_{i i}$ can and should be verified by stochastic simulation techniques. Such a verification was done in cases for the commonly used models in microbiology. For most cases it was shown that for moderate sample sizes $N$ the sampling variances of the parameter estimates are well approximated by formula (9).

Note that the precision of the estimates can always be decreased by increasing the sample size $N$, which yields a 'smaller' covariance matrix and smaller variances of the least squares estimates. However, in practice the sample size is usually fixed, due to cost considerations of each additional experiment. To improve the quality of the estimates or, from a different point of view, to reduce the number of experiments needed to obtain the estimates with a given accuracy we note that the variances of the estimates $\hat{\theta}_{i(N)}$ and the covariance matrix of the vector $\hat{\theta}_{(N)}$ also depends on the given design $\xi$, which determines the relative proportion of total observations to be taken at the experimental conditions $t_{1}, \ldots, t_{n}$ and its location. Therefore the question arises if one can find a design in some optimal way.

Example 2.4. Consider a special case of the exponential regression model in Example 2.1, that is

$$
\eta(t, \theta)=e^{-\theta t}
$$


where $t \in[0,10]$ and assume that the unknown parameter is given by $\theta^{*}=0.05$ and that $\sigma^{2}=1$. The length of the confidence interval is given by

$$
\frac{2 u_{1-\frac{\alpha}{2}}}{\sqrt{N}} \sqrt{\left(M^{-1}\left(\xi, \theta^{*}\right)\right)_{11}}
$$

where the Fisher information is given by

$$
M(\xi, \theta)=\sum_{i=1}^{n} \omega_{i}\left(\frac{\partial}{\partial \theta} \eta\left(t_{i}, \theta\right)\right)^{2}=\sum_{i=1}^{n} \omega_{i} t_{i}^{2} e^{-2 t_{i} \theta} .
$$

Assume that a $95 \%$ coverage probability is desired and that $N=100$ are available. If observations are taken according to the uniform design (i.e. $t_{i}=i / 10 ; \omega_{i}=1 / 100 \quad i=1, \ldots, n=100$ ) the length of the confidence interval is approximately

$$
2 \cdot 0.196 \cdot 0.2481 \approx 0.0972
$$

On the other hand, assume that the experimenter takes the 100 observation only at $n=5$ different experimental conditions uniformly, that is $t_{i}=2 i, \omega_{i}=1 / 5 \quad i=1, \ldots, 5$, then the length of the confidence interval is

$$
2 \cdot 0.196 \cdot 0.2244 \approx 0.0879 .
$$

Thus we obtain a reduction of the length of the tolerance region simply by sampling at different experimental conditions. A further reduction of the length could be obtained by sampling at only two different experimental conditions, e.g. $t_{1}=10, t_{2}=20, \omega_{1}=\omega_{2}=1 / 2$. In this case the length of the interval would be

$$
2 \cdot 0.196 \cdot 0.1483 \approx 0.0581
$$

and the question arises if the experimental conditions can be chosen such that the length of the confidence interval is minimal. This is precisely the problem of finding an optimal experimental design, which will be described and discussed next.

In general an optimal design maximizes or minimizes the value to a given function of the Fisher information matrix (see, f. e., Silvey, 1980). However this matrix depends on the vector $\theta^{*}$, which is unknown. To overcome this difficulty a simple and often rather efficient approach is to substitute an initial guess $\theta^{0}$ for the unknown value $\theta^{*}$. The corresponding designs are called local optimal designs

(Chernoff, 1953). The most popular criteria of optimality are $D$-, $c$ - and $E$-criteria (Pukelsheim, 1993). These criteria have appeared in the literature on optimal design for microbiological nonlinear models and we review some of them for the sake of completeness.

A design is called local $D$-optimal if it maximizes the quantity

$$
\operatorname{det} M\left(\xi, \theta^{0}\right)
$$


where $\theta^{0}$ is a given initial value for the true parameter vector. It can be shown that an optimal design with respect to this criterion yields approximately a minimal value for the volume of the tolerance ellipsoid of the estimates (see, f. e., Karlin, Studden, 1966, ch. X). In addition, due to the famous equivalence theorem introduced in Kiefer, Wolfowitz (1960), this design minimizes an approximation of the worst variance of the predicted response $\eta\left(t, \hat{\theta}_{N}\right)$ over the interval $[0, T]$, that is

$$
\max _{t \in[0, T]} \mathcal{D}\left(\eta\left(t, \hat{\theta}_{N}\right)\right)
$$

For a given vector $c \in \mathbb{R}^{m+1}$ a design is called local $c$-optimal if it minimizes the variance

$$
\mathcal{D}\left(c^{t} \hat{\theta}_{N}\right)=\frac{\sigma^{2}}{N} c^{T} M^{-1}\left(\xi, \theta^{o}\right) c
$$

of the estimate of a given linear combination of the parameters $\theta^{T} c=\sum_{i=0}^{m} \theta_{i} c_{i}$. The important choices for the vector $c$ are contrasts, where the sum of the components of the vector $c$ is 0 and unit vectors, i.e.

$$
c=e_{i}=(\underbrace{0, \ldots, 0}_{i}, 1,0, \ldots, 0)^{T} .
$$

In the latter case a $c$-optimal design minimizes the variance of the least squares estimate for the $i$ th parameter (under the condition that $\theta^{*}=\theta^{0}$ ). Such a criterion was used in Example 2.4, where the interest was the length of a particular confidence interval. If the experimenter has particular interest in several linear combinations of the parameters a local E-optimal design seems to be appropriate. This design maximizes the minimum eigenvalue

$$
\lambda_{\min }\left(M\left(\xi, \theta^{0}\right)\right)
$$

of the Fisher information matrix. A standard argument from linear algebra shows that this maximization is equivalent to the minimization of the maximum eigenvalue of the inverse Fisher information matrix $M^{-1}\left(\xi, \theta^{0}\right)$ or to the minimization of the worst variance among all estimates $c^{T} \hat{\theta}_{N}$ for the linear combinations $c^{T} \theta$ with a given norm $c^{T} c=1$.

Another important criterion used in microbiological studies is the modified $E$-criterion

$$
\frac{\lambda_{\min }\left(M\left(\xi, \theta^{0}\right)\right)}{\lambda_{\max }\left(M\left(\xi, \theta^{0}\right)\right)},
$$

and an optimal experimental design with respect to this criterion makes this quantity as small as possible.

For many models used in microbiology it has been proved either by mathematical rigorous arguments or by intensive numerical experiments that the number $n$ of different experimental conditions for a local optimal design is equal to the number of parameters in the nonlinear regression model, i.e.

$$
n=m+1
$$


It is also usually the case that the local optimal designs advise the experimenter to take observations at the maximal possible value $T$ for the explanatory variable. Moreover, if $n=m+1$ the optimal relative proportions of total observations to be taken at the experimental conditions $t_{0}, \ldots, t_{m}$ can usually easily be determined as functions of the points $t_{0}, \ldots, t_{m}$ (see Pukelsheim and Torsney, 1991), where the particular function depends on the optimality criterion under consideration. For example, for the case of the $D$-optimality criterion these functions are constant and all equal, i.e. $\omega_{i}=1 /(m+1), i=0,1, \ldots, m$ (see, f. e., Fedorov, 1972) and similar formulas are available for the $c$-and $E$-optimality criterion [see Pukelsheim and Torsney (1991)]. These facts are very helpful for the determination of local optimal designs, because they reduce the dimension of the optimization problem.

If we have found a local optimal design we should study its sensitivity with respect to the choice of the initial value $\theta^{0}$. This can be done by calculating the relative efficiency of a local optimal design at the point $\theta_{0}$ with respect to the local optimal designs at other points from a given set. For example, in the case of $D$-optimality criterion one usually considers the quantity

$$
I(\xi)=\min _{\theta \in \Omega} I(\xi, \theta),
$$

where $\Omega$ is a given set of possible values for the unknown parameter $\theta^{*}$,

$$
I(\xi, \theta)=\left(\frac{\operatorname{det} M(\xi, \theta)}{\operatorname{det} M\left(\xi_{\theta}, \theta\right)}\right)^{1 /(m+1)},
$$

is the $D$-efficiency of the given design $\xi$ with respect to the local $D$-optimal design $\xi_{\theta}$ under the assumption that $\theta^{*}=\theta$ is the 'true' parameter. The value $I(\xi, \theta)$ indicates how many more observations will be needed under the design $\xi$ to obtain a given accuracy with respect to the $\operatorname{design} \xi_{\theta}, \theta=\theta^{*}$. If it can be shown that the accuracy of the local optimal design for the choice $\theta^{0}$ is not too sensitive with respect to the choice of the initial value $\theta^{0}$ the application of local optimal designs is well justified. For most models in microbiology it was shown that local optimal designs are often robust with respect to the choice of the initial parameter $\theta^{0}$ (see f.e. Bezeau, Endrenyi, 1986; Dette et. al., 2003) and for this reason we will mainly concentrate on local optimal designs in this paper.

However, if the dependence of the optimal design on the nonlinear parameter is more severe, or if the experimenter has no prior knowledge about the location of the unknown parameters, some care is necessary with the application of local optimal designs. In this case some more sophisticated optimality criteria are required for the construction of efficient and robust designs, which will be briefly mentioned here for the sake of completeness. We restrict ourselves to the $D$-optimality criterion, but the idea for other types of criteria is very similar.

(i) If one can split the whole experiment into several stages with $N_{1}, \ldots, N_{r}$ observations taken at each stage, then it can be useful to use a so called sequential experimental design (see Fedorov, 1972; Silvey, 1980). In this approach the estimate constructed at the previous stage 
can be used to construct a design for a current stage. For example, for the $D$-criterion we start start with an initial guess for the unknown parameter, say $\theta^{0}$ and take $N_{1}$ observations according to the local $D$-optimal design (i.e. the design, which maximizes $\left.\operatorname{det} M\left(\xi, \theta^{0}\right)\right)$ to obtain the least squares estimate $\hat{\theta}_{\left(N_{1}\right)}$. In the second stage this estimate is used as preliminary guess and a new design is determined by maximizing the determinant $\operatorname{det} M\left(\xi, \theta_{\left(N_{1}\right)}\right)$ to obtain the $N_{2}$ observations for the second stage of the experiment. The least squares estimate from the first $N_{1}+N_{2}$ observations, say $\hat{\theta}_{\left(N_{1}+N_{2}\right)}$ is then used as preliminary guess for the third stage and the next $N_{3}$ observations are taken according to the design maximizing the determinant $\operatorname{det} M\left(\xi, \hat{\theta}_{\left(N_{1}+N_{2}\right)}\right)$. This procedure is continued for all $r$ stages to obtain the least squares estimate $\hat{\theta}_{(N)}=\hat{\theta}_{\left(N_{1}+\ldots+N_{r}\right)}$ from the total sample. It should be noted that inference from a sequential design is not easy (see e.g. Ford and Silvey, 1980; Ford, Titterington and Wu, 1985; Wu, 1985 or Woodroofe and Coad, 2002). Moreover, there exist many experiments, where observations cannot be taken at several stages.

(ii) In order to obtain a non-sequential design, which is less sensitive with respect to the choice of the parameter $\theta^{0}$ several authors proposed to construct a design maximizing the value $I(\xi)$ for a given set $\Omega$ with respect to the choice of the experimental design. Such designs are called maximin efficient designs (see Müller, 1995). Maximin efficient designs for the $D$ - and E-optimality criterion were constructed in Dette and Biedermann (2003) and Dette, Melas, Pepelyshev (2003a) for the Michalis-Menten model, respectively. Further examples can be found in Biedermann, Dette and Pepelyshev (2004), who considered a compartmental model, and Dette, Melas and Wong (2004), who discussed this type of design in the Hill model. Some more general results for this type were obtained by Dette, Haines and Imhof (2003a,b). We finally note that the determination of maximin efficient designs is a substantially more difficult problem compared to the problem of determining local optimal designs.

(iii) A different robust alternative is to assume sufficient knowledge of $\theta$ to specify a prior distribution for this parameter and to average the respective optimality criteria over the plausible values of $\theta$ defined by the prior. This leads to so-called Bayesian optimality criteria [see e.g. Chaloner and Larntz (1989) or Chaloner and Verdinelli (1995)] and we will discuss this approach separately in Section 5.

\section{Applications of optimal experimental design in micro- biology.}

The parameters of models for describing quantitative data and predicting outcomes of microbiological processes often have special names and their values play an important role in the analysis of experimental results. Most of the models, which describe microbial growth are non-linear first order differential equations in several variables. Variables are related by the stoichiometric co- 
efficients and reflect mass-balances equations (Pirt, 1975; Howell, 1983; McMeekin, Ross, 2002). Additionally at some time point (usually at initial point), all coefficients and variable definitions are specified. One of the important stages of the mathematical modelling application is a comparison of the model predictions with the real experimental data and identification of the model parameters and optimal experimental designs are very useful at this stage.

The problem of identification of the model parameters concerns two important questions. The first question is how well the mathematical model reflects the real microbiological process. This question is a very difficult one; the answer always depends on the level of details of the research. For example if one will consider degradation of organic pollutants, for example nitroaromatic compounds, by a community of microorganisms with complex and unknown structure (let us imagine activated sludge), then it should be sufficient to choose an unstructured growth model such as the classical Monod model or some of its modifications such as Andrews or Haldane use to describe this microbiological process (Ellis et al., 1996; Knightes, Peters, 2000; Goudar, Ellis 2001). On the other hand, if one will consider a much more "simple" microbiological process, such as utilization of glucose by Escherichia coli, we would find that the Monod model is not a realistic tool for describing this process and it is necessary to use models with more complicated structure (Ferenci, 1999). One of the important methods for examining if a particular model is adequate for a given microbiological process is the comparison of the model predictions with experimental data. Unfortunately, a good fit of the model is only a necessary but not a sufficient condition to be confident that the selected model is realistic. It is not difficult to identify models which yield a good fit to experimental data but are not realistic (Hopkins, Leipold, 1996). The model selection is not a formal procedure and it requires simultaneous theoretical and experimental biological efforts.

If the first question is answered then the second problem is the identification of model parameters and the question of the efficiency of an experiment arises. This problem plays the central role in our paper. The following questions should be answered:

1) Is it possible to determine parameters of the mathematical model which describe the microbiological process using the particular experimental procedure? And if that answer is 'no' can we find such scheme of measurements for which the answer is 'yes'?

2) Is it possible to make a relatively small number of experiment replications and measurements without loosing information?

Without a clear understanding that the model parameters are unique and their values are realistic, it is not possible to use them as characteristics of a microbiological process. Ignoring these important questions leads to mistakes and unsuccessful application of mathematical models for the analysis and prediction of microbiological processes. Although the mathematics cannot guarantee that the necessary data is obtainable it can be extremely useful in the sense that it can significantly reduce the number of necessary measurements and experimental replications. A good experimental design can therefore help to economize laboratory time and expenses. 
The present section consists of two parts. First we will consider ideas of optimal experimental design using the Monod model as an example. Next we will review some literature on applications of optimal design techniques to microbiological models, which are not related to the Monod model.

\subsection{The Monod model}

The Monod model can describe several important characteristics of microbial growth in a simple periodic culture of microorganisms. This model was proposed by Nobel Laureate J. Monod more than 50 years ago and it is one of the basic models for quantitative microbiology (Monod, 1949, Ferenci, 1999). After several decades of intensive studies many limitations of this model as well as restrictions of its applications are very well known (Pirt, 1975; Baranyi, Roberts, 1995; Ferenci, 1999). At the same time many modifications of this model have been proposed in some specific cases (Ellis et al., 1996; Fu, Mathews, 1999; Schirmer et al., 1999; Vanrolleghem et al., 1999). The Monod model is still used very often without any modifications, especially in such fields as environmental and industrial microbiology. For example, it is the most common model for describing the dynamics of organic pollutant biodegradation (Blok, Struys, 1996; Knightes, Peters, 2000; Goudar, Ellis, 2001). At the same time the similarity of the Monod model and the MichaelisMenten equation provides a very wide application of this model type throughout biological and biomedical sciences. This type of equation is very often used in biochemistry, plant physiology, biophysics and pharmacology.

The Monod model (Monod, 1949, Pirt, 1975) for periodic culture (batch) experiments may be presented as a first order differential equation:

$$
\eta^{\prime}(t)=\mu(t) \eta(t)
$$

where

$$
\begin{gathered}
\mu(t)=\theta_{1} \frac{s(t)}{s(t)+\theta_{2}} \\
s(t)-s_{0}=\frac{\eta_{0}-\eta(t)}{\theta_{3}}
\end{gathered}
$$

Here $s_{0}=s(0)$ and $\eta_{0}=\eta(0)$ are given initial conditions, i.e., initial concentrations of the consuming substrate and microbial biomass, respectively. Three parameters characterize microbial growth. Each parameter has its own traditional notation and name: $\theta_{1}$ is called the maximal specific growth rate usually denoted by $\mu_{\max }, \theta_{2}$ the saturation (affinity) constant denoted by $K_{s}$, $\theta_{3}$ the yield coefficient usually denoted by $Y$. The variable $t$ represents time, which varies in the closed interval $[0, T]$. Typical minimum values of $T$ are several hours for optimal microbiological media, whereas the maximum is one year or more for specialized groups of

microorganisms. All three parameters, initial conditions and variables are positive because of natural biological conditions. This differential equation can be easily integrated as indicated in the previous section. 
The general meaning of this model is that the rate of microbial growth (i.e. change of microbial biomass) is equal to the current biomass value multiplied by the specific growth rate. Specific growth is a function of the concentration of nutrients with two parameter characteristics for a given microorganism (maximum specific growth rate and saturation constant or Michaelis-Menten constant). At the same time increase of microbial biomass is proportional to the decrease of nutrients where the coefficient of proportionality is the Yield coefficient $Y$.

The parameter estimation problem for the Monod and Monod-type models has been considered many times with different mathematical and biological assumptions (Aborney, Williamson, 1978; Holmberg, 1982; Baltes, 1994; Vanrolleghem et al., 1995). Some of the results are more theoretical and other are more related to a specific experimental procedure. Usually the best technique for parameter identification are chosen based on the experimental procedure. For Monod-type models experimental procedures vary greatly dependent on measurements technique and reactor type. There are several main variants of simple batch experiments, as indicated below:

1) The batch-type experiment where it is possible to make only measurements of the biomass value or some parameter which is related to biomass activity, for example oxygen uptake or carbon dioxide production (Blok, Struys, 1996; Vanrolleghem et al., 1995). The structural identifiability of the parameters in the Monod model for this case was analyzed by Chappel and Gofrey (1991).

2) Experiments where it is possible to make simultaneous measurement of the consumed substrate and the biomass concentration. An example of this type of experiment is the investigation of the biodegradation of the volatile hydrophobic organic compounds such as phenanthrene (Guha, Jaffe, 1996), where parameters in the Monod model were determined by maximum likelihood estimation from direct measurements of the phenanthrene concentrations and carbon dioxide production. Applications of the least squares estimation techniques for this case were considered from a theoretical perspective in several publications (Aborney, Willianson, 1972; Holmberg,1982; Saez, Rittmann, 1992)

To discuss the main ideas of optimal experimental design let us consider the first type of experiment. For example, we are investigating biodegradation of some organic pollutant, which is the unique source of organic and energy for the growing microorganisms (Sommer et al., 1995) and we can only make measurements only of the biomass of microorganisms at different time points (or for example respiratory activity). Then the results of the experiment is a table $\left\{t_{i}, x_{i}\right\}$ with two rows; the first row is the time of the measurement $\left\{t_{i}\right\}$, and the second is the value a microbial biomass $\left\{x_{i}\right\}$. The first column of this table is called experimental design; it will be denoted as $\xi$, and to be more mathematically precise we can say that it is a discrete probabilistic measure with equal weights [see Section 2]. It is obvious that before we start an experiment it is important to decide when we will make our measurements, but we should also leave open the possibility of changing the experimental design after the experiment has started. To illustrate the optimal design approach artificial experimental data sets will be used. This approach is very often used 


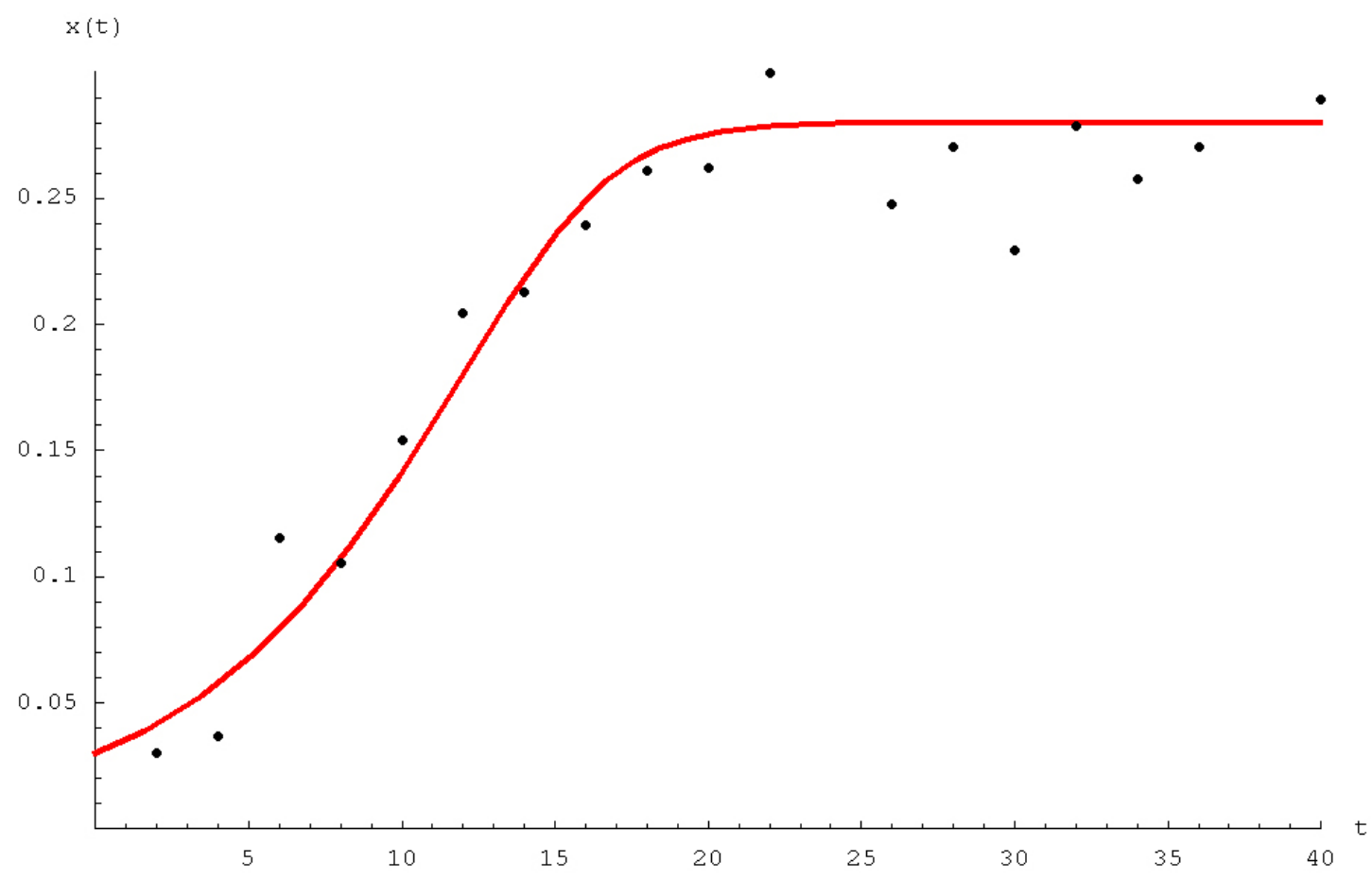

Figure 1: Artificial experimental data and the plot of the Monod solution (20 uniform points experimental design with normal distributed experimental errors, dispersion 0.02).

with Monte-Carlo simulations as a tool for the analysis of regression models (Holmberg,1982; Ellis et al., 1996; Melas, Strigul, 1999; Poschet et al., 2003). Let us decide to make measurements every 2 hours up to 40 hours; then our experimental design is the $\{0,2,4,6 \ldots 40\}$. A typical example of data is illustrated in Figure 1. The experimental data will be simulated artificially. Let us fix some parameter set called 'true' value $\theta^{*}$, for example $\theta_{1}^{*}=0.25 ; \theta_{2}^{*}=0.5 ; \theta_{3}^{*}=0.25$ (this set is the average of several experimental results, see Pirt, 1975). The Monod model solution is calculated for this parameter $\theta^{*}$ with the initial conditions $s_{0}=1$ and $\eta_{0}=0.03$. Consider the values of the solution of the Monod model at the points of our experimental design, $\eta_{i}=\eta\left(t_{i}, \theta\right), i=1, \ldots, 20$. At this stage the ideal set of experimental data is constructed. It is such a set of experimental measurements which was obtained if there were no experimental errors in both measurements and time of measurements. 


\begin{tabular}{lllllll}
\multicolumn{4}{c}{ uniform design } & \multicolumn{4}{c}{ D-optimal design } \\
& $\widehat{\theta}_{1}\left(\mu_{\max }\right)$ & $\widehat{\theta}_{2}\left(K_{s}\right)$ & $\widehat{\theta}_{3}(Y)$ & $\widehat{\theta}_{1}\left(\mu_{\max }\right)$ & $\widehat{\theta}_{2}\left(K_{s}\right)$ & $\widehat{\theta}_{3}(Y)$ \\
1 & 0.1645 & 0.0795 & 0.2441 & 0.2545 & 0.5097 & 0.2492 \\
2 & 0.3087 & 0.9239 & 0.2557 & 0.2541 & 0.5123 & 0.2455 \\
3 & 0.2520 & 0.5295 & 0.2547 & 0.2452 & 0.4814 & 0.2556 \\
4 & 0.1497 & 0.0477 & 0.2565 & 0.2661 & 0.5725 & 0.2519 \\
5 & 0.1639 & 0.0597 & 0.2434 & 0.2451 & 0.4533 & 0.2487 \\
6 & 0.1851 & 0.2446 & 0.2562 & 0.2690 & 0.5430 & 0.2521 \\
7 & 0.2007 & 0.2456 & 0.2342 & 0.2489 & 0.5224 & 0.2568 \\
8 & 0.2937 & 0.7057 & 0.2624 & 0.2389 & 0.4631 & 0.2519 \\
9 & 0.3251 & 0.9019 & 0.2397 & 0.2569 & 0.5313 & 0.2534 \\
10 & 0.2255 & 0.3607 & 0.2584 & 0.2510 & 0.4848 & 0.2458 \\
\hline Average & 0.2269 & 0.4099 & 0.2505 & 0.2530 & 0.5074 & 0.2511 \\
CD & 0.0647 & 0.3379 & 0.0094 & 0.0093 & 0.0371 & 0.0038
\end{tabular}

Table 1. Replications of least squares estimates of the parameters in the Monod model with a uniform design (20 equidistant distributed points in the interval $[0,40]$ and a local D-optimal design, which uses only three different experimental conditions). The 'true' parameters are $\theta_{1}^{*}=0.25, \theta_{2}^{*}=0.5, \theta_{2}^{*}=0.25$, while the experimental errors are generated from a normal distribution with variance $\sigma^{2}=0.02$.)

But the most important property of real experimental data is the existence of random error in measurements. In real life there are never precise measurements; there are errors in the biomass measurements (for any experimental technique) and sometimes significant errors in the time of measurements. Suppose that those errors are random and they are normally distributed with some fixed values of dispersion. For example suppose the variance of random errors equals 0.02. Then the table of the artificial experimental data using the values of the microbial biomass are the numbers randomly taken from this normal distribution with these values as averages, i.e. $\eta_{i}^{*}=\eta_{i}+\varepsilon_{i}$, where $\varepsilon_{i} \in N(0 ; 0.02)$. Assume that we can repeat the experiment several times, say, $k$ times, and hence we will have $k$ independent sets of the experimental data $\left\{\eta_{i}^{*}\right\}_{j}, i=1, \ldots, 20$, $j=1, \ldots, k$. The number of replications $k$ is a very important characteristic of any experiment scheme, of course we would like to make as few experimental replications as possible without loss of information. The experimental optimal design approach is useful in making $k$ minimal.

Let us forget that we know the 'true' parameter' values $\theta^{*}$ and assume that after the experiment we have the results of the biomass measurements $\left\{\eta_{i}^{*}\right\}_{j}$. Then our aim is to estimate the Monod model parameters from these data. This situation is very close to real problems (Knightes, Peters, 2000; Schirmer et al., 1999). What do we have at this stage? First, we know that the experiment is very well described by the Monod model (note that in real applications the specification of a nonlinear regression model is a nontrivial problem). Second, we have experimental data sets with some realistic replication number $k$, say, $k=5$.

The problem of determining the least squares estimate in a non-linear regression model (that is the set of parameter estimates that minimizes the sum of squared residuals in (7)) is a nonlinear 
optimization problem, which can be solved by standard algorithms, for example the Gauss-Newton method. In the following we denote the set of Monod parameter estimates as $\widehat{\theta}=\left\{\widehat{\theta}_{1}, \widehat{\theta}_{2}, \widehat{\theta}_{3}\right\}$ and the parameter estimates obtained from the $k$ experiments by $\widehat{\theta}^{1}, \ldots, \widehat{\theta}^{k}$. How do we know that the obtained estimation $\widehat{\theta}$ is close enough to the 'true' parameters $\theta^{*}$ ? Actually, we can say only that the non-linear least squares estimate are good enough asymptotically, i.e. consistent and asymptotically normally distributed if the sample size is sufficiently large. [see Jennrich (1969) and Dette et al. (2003)]. Unfortunately mathematics cannot guarantee that any of the estimates $\left\{\widehat{\theta}^{j}\right\}_{j=1, \ldots k}$ will be close enough to the 'true' parameter $\theta^{*}$ when the sample size is not very large. As we can see from Table 1 , the ten independent estimates of $\widehat{\theta}$ do not provide precise estimations of $\theta^{*}$ (the question of what is a precise estimate is also very important; suppose at most $10 \%$ error is the criterion of good estimation). The estimates of $\widehat{\theta}_{1}, \widehat{\theta}_{2}$ are not close to the 'true' values $\theta_{1}^{*}$ and $\theta_{2}^{*}$ in the presented replications and the standard deviation of them is large. At the same time the estimate $\widehat{\theta}_{3}$ determines the component $\theta_{3}^{*}$ with sufficient accuracy practically in each replication. For better understanding of this result let us make a correlation analysis of the results of 100 independent replications $(k=100)$. It is important that the estimators $\widehat{\theta}_{1}$ and $\widehat{\theta}_{2}$ are closely correlated with the correlation coefficient 0.99 , at the same time the estimator $\widehat{\theta}_{3}$ is not correlated with the other two estimates. The two dimensional plot (Figure 2 ) of $\widehat{\theta}_{1}$ and $\widehat{\theta}_{2}$ can be considered as the level surface of the non-linear square estimation, as long as the values of the $\min \sum\left(\eta_{i}^{*}-\right.$ $\left.\eta\left(t_{i}, \theta\right)\right)^{2}$ are very close in those experiments (table 1 ). It is interesting to examine the three dimensional plot of $\sum\left(\eta_{i}^{*}-\eta\left(t_{i}, \theta\right)\right)^{2}$ for varying values of $\theta_{1}$ and $\theta_{2}$ (Figure 3 ). The lowest level of the sum of squares is located on the line at the bottom; it looks very similar to a ravine. We can conclude that linear dependence of $\widehat{\theta}_{1}$ and $\widehat{\theta}_{2}$ is a serious problem for a precise estimation of the parameters $\theta_{1}^{*}$ and $\theta_{2}^{*}$ for realistic sample sizes. For the Monod type of nonlinearity, i.e. for the form of the Michaelis-Menten function this problem was described many times by different groups of researchers (Holmberg, 1982; Magbanua et al., 1998). It was shown that in the considered experimental conditions it is not possible to identify parameters of the Monod model (Holmberg, Ranta, 1982). 


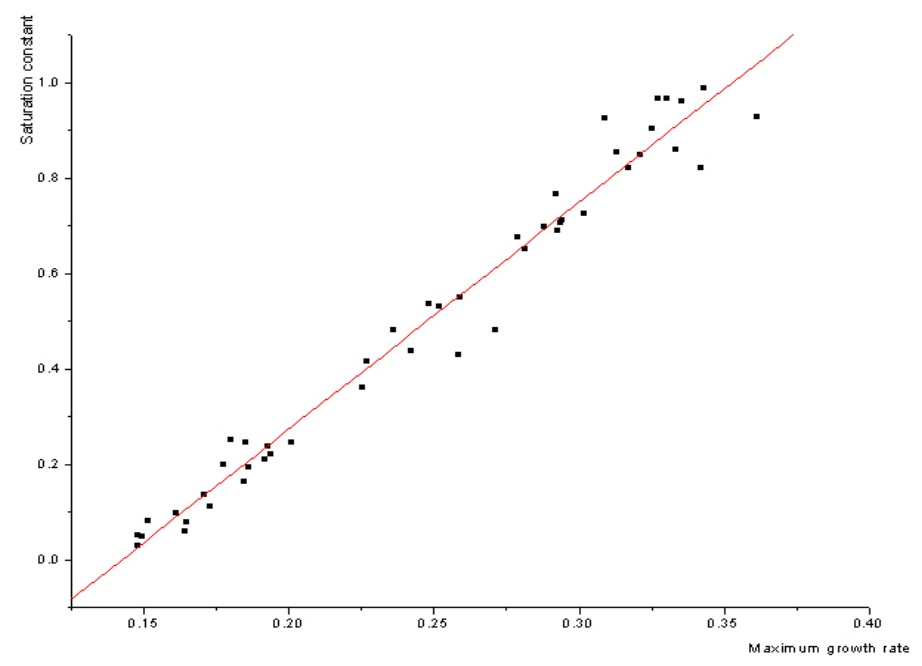

Figure 2: Scatter plot of the estimations of the Monod model parameters: Maximum specific growth rate and saturation constant, $n=100, r=0.987, p<0.0001$.
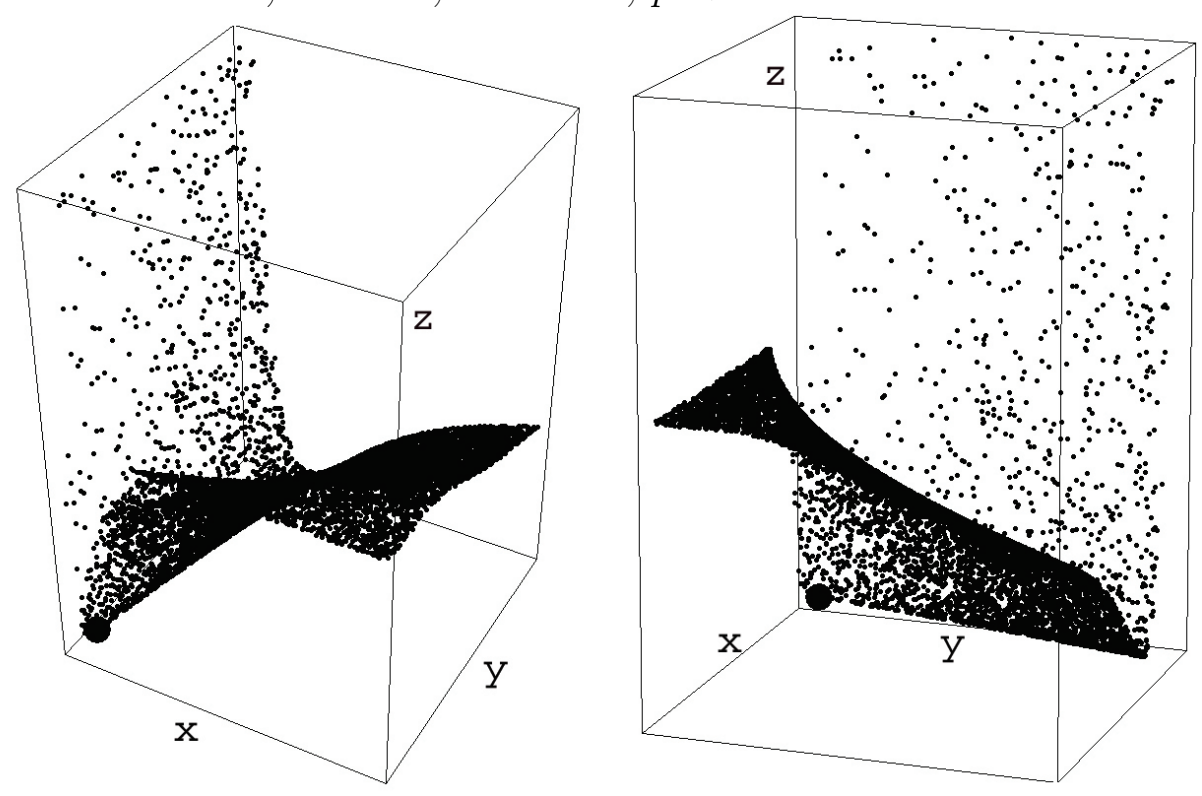

Figure 3: Regression surface for one artificial data set (large point is the true parameters value). 
Since the problem of the correlation between $\widehat{\theta}_{1}$ and $\widehat{\theta}_{2}$ plays a central role in there considerations it is useful to obtain an elementary interpretation of this problem. In Figure 4,5 and 6 we represent results of the solution of the Monod differential equation for a variation of the model parameters. As we can see that $\theta_{3}$ mostly determines the value of the horizontal asymptote of the solution $\eta(t)$ and that $\theta_{1}, \theta_{2}$ determine the bottom and upper bends of the solution. The lower bend is called the exponential phase of microbial growth and the upper bend is the retardation phase (Monod, 1949). In some sense the change of the maximal specific growth rate $\theta_{1}$ (for example increase) can be compensated by the opposite change of the Michaelis-Menten constant (decrease) and the graph of the solution will be very similar. Hence, the correlation between $\widehat{\theta}_{1}$ and $\widehat{\theta}_{2}$ means that if we plot such solutions of the Monod equation with very different values of $\theta_{1}, \theta_{2}$ than they will have the same level of non-linear least squares estimation to the experimental points (Figure 7). It is possible to consider several alternative approaches to deal with this problem:

(i) One could use a different mathematical model for the analysis of the data (but obviously in this case the Monod model is the correct chosen model)

(ii) One could increase the number $k$ of experimental replications (but it is not known how many replications will guarantee good parameter estimates and each replication causes additional expenses)

(iii) One could improve the precision of the experimental measurements to reduce the dispersion of the measurement errors (this is not always possible and may require additional expensive equipment)

(iv) One could apply another mathematical estimation technique, for example maximum likelihood estimation, which is sometimes better than the simple non-linear least square regression technique (Sommer et al., 1995; Knightes, Peters, 2000), or an estimation algorithm based on the Lyapunov's stability theory (Zhang, Guay, 2002).

(v) One could use an optimal experimental design to define the different experimental conditions (there is no guarantee of improvement by a design obtained from asymptotic theory, but if this approach will be efficient it will have more advantages compared to the previous approaches because it does not cause additional costs).

Let us concentrate on the application of optimal experimental design and describe a way to construct an experimental design such that parameters will be identified very well from a minimal number of experimental measurements $n$ and a minimal number $k$ of replications of the experiment. This approach for Monod-type models was developed theoretically (Munack, 1989; Baltes, 1994; Versyck et. all 1998; Dette et al., 2003) and applied in several types of experiments (Vanrolleghem et al., 1995; Ossenbruggen et al., 1996; Merkel et al., 1996). First of all it is known that the parameters of the Monod model can be identified (see Dette et. al. 2003; Petersen et al., 


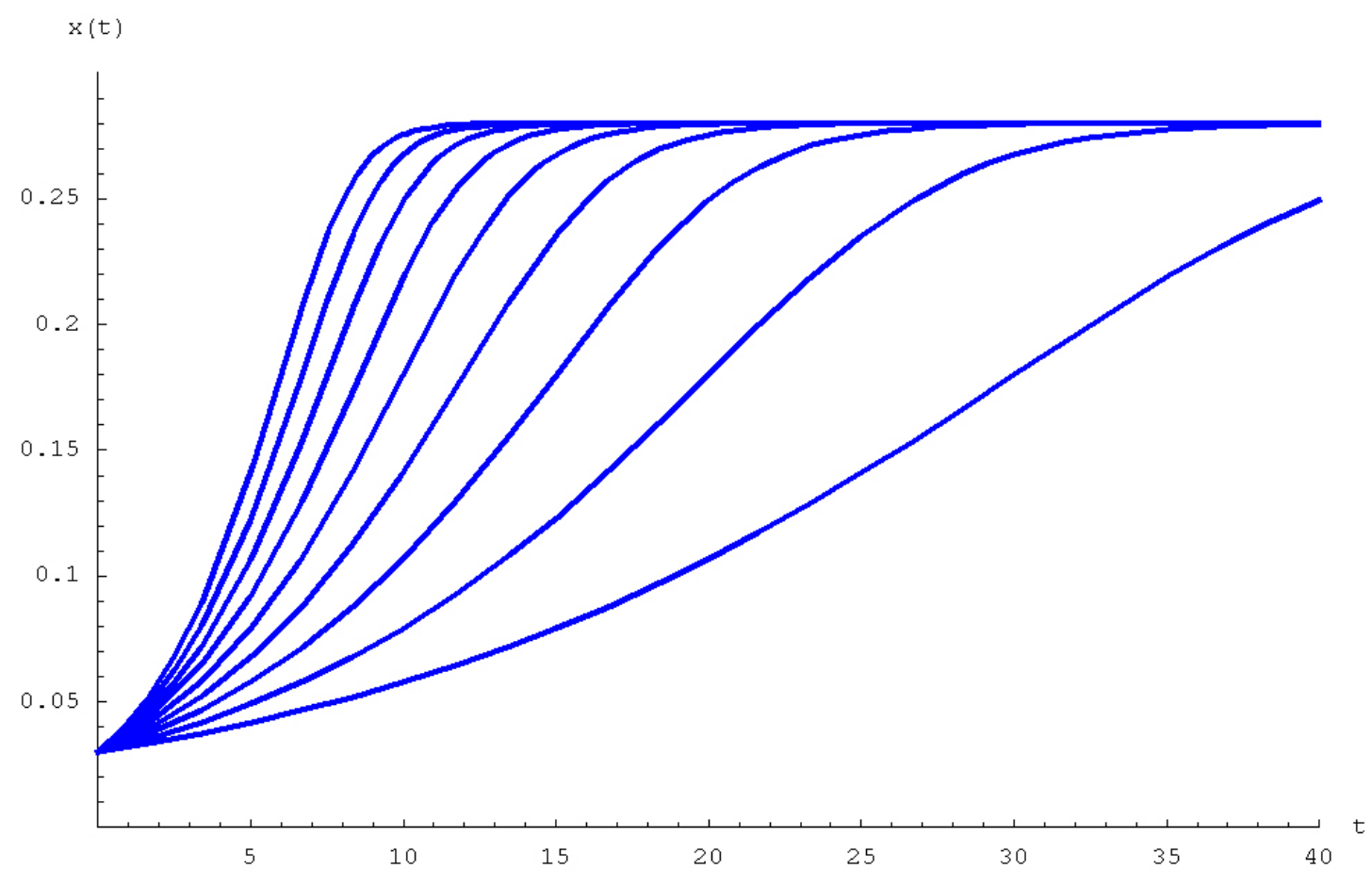

Figure 4: Effect of the maximum specific growth rate on the solution of the Monod equation (maximal specific growth rate was changed from 0.1 to 0.5 with step 0.05 , other parameters were fixed $(0.5,0.25)$; initial conditions $\left.x_{0}=0.3, S_{0}=1\right)$.

$x(t)$

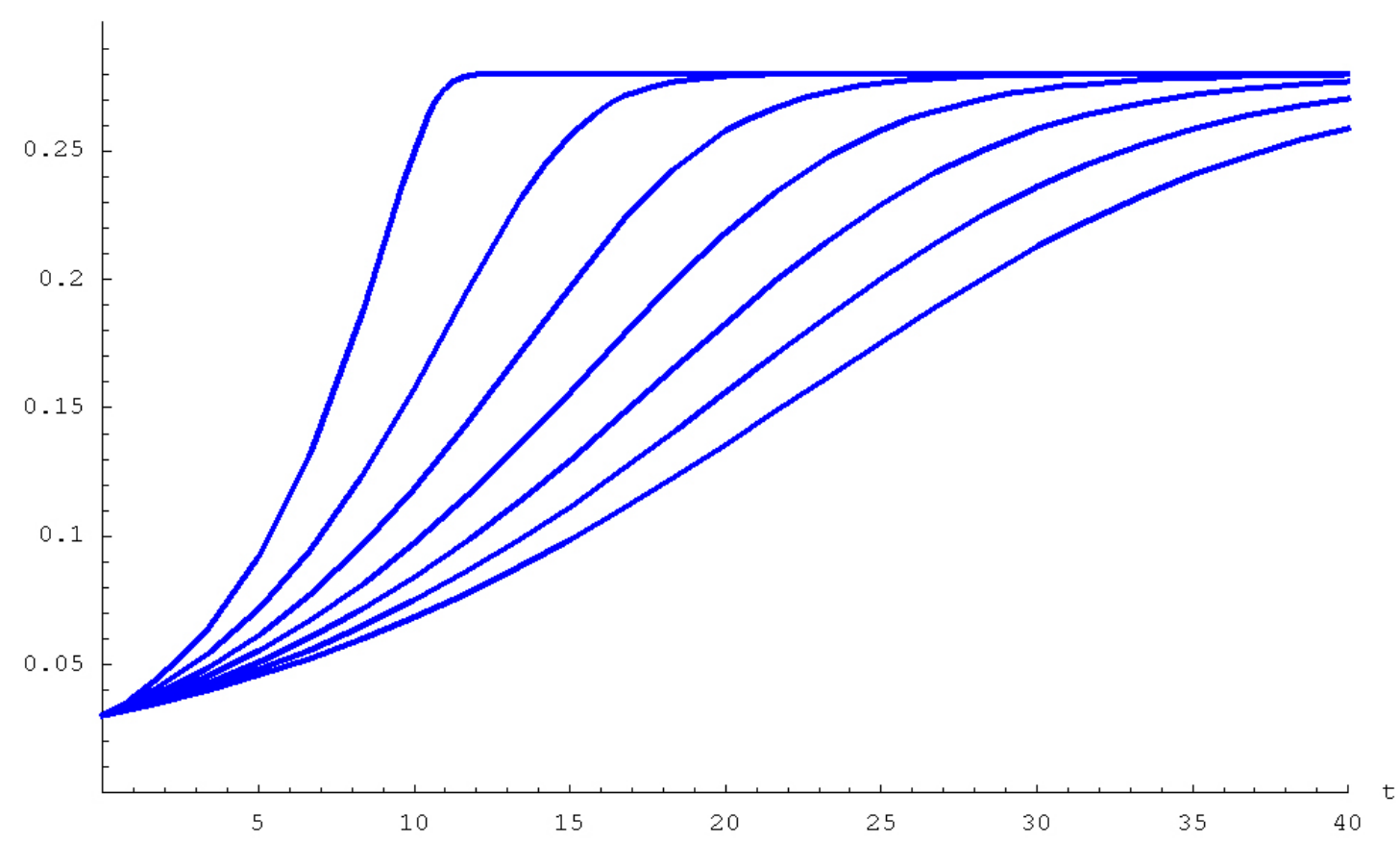

Figure 5: Effect of the Michaelis-Menten constant on the solution of the Monod equation (the Michaelis-Menten constant was changed from 0.1 to 1.9 with step 0.3 , other parameters were fixed (0.25, 0.25); initial conditions $\left.x_{0}=0.3, S_{0}=1\right)_{20}$ 


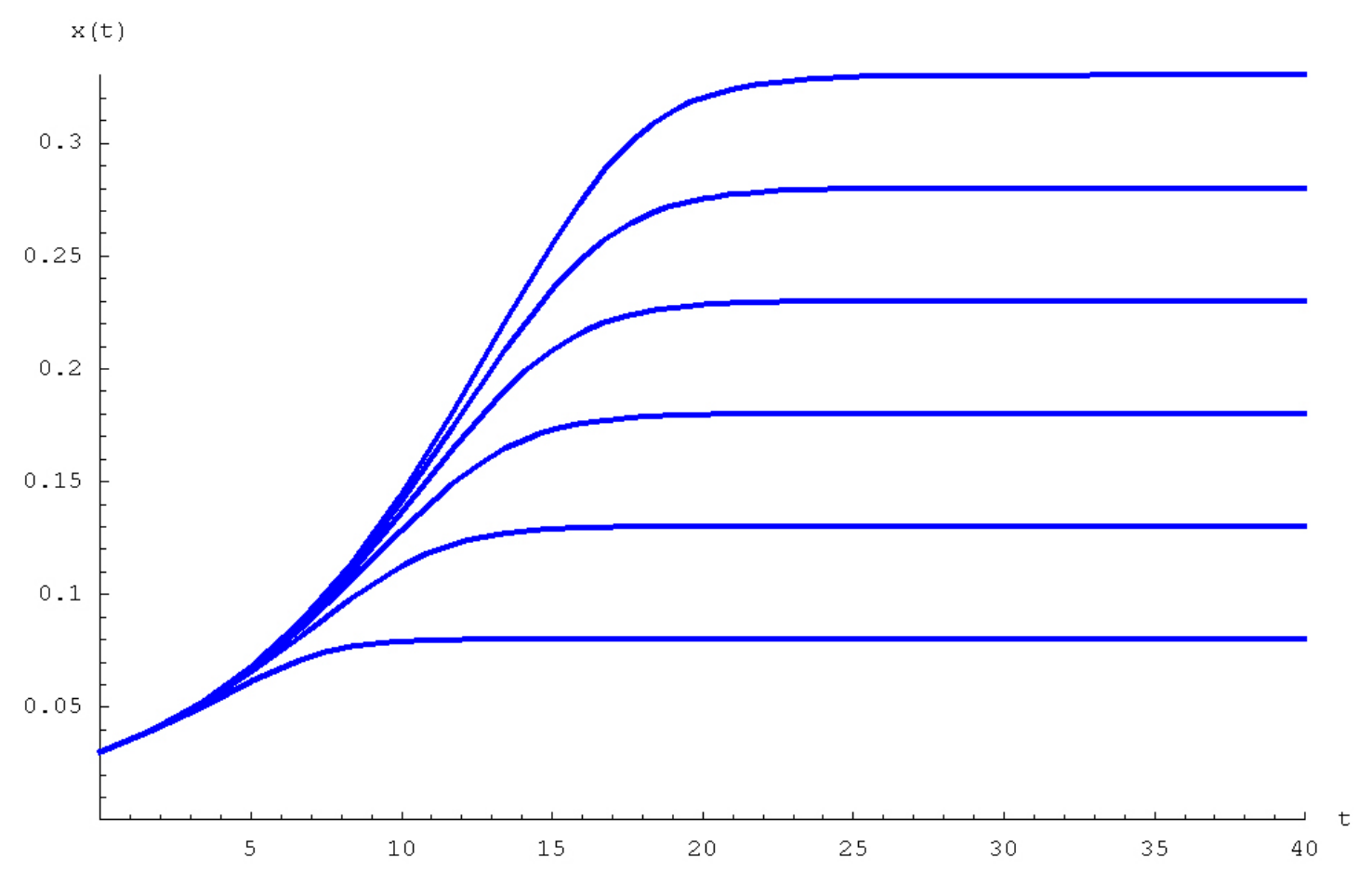

Figure 6: Effect of the Yield coefficient on the solution of the Monod equation (the Yield coefficient was changed from 0.05 to 0.3 with step 0.05 , the other parameters were fixed $(0.25,0.5)$; initial conditions $x_{0}=0.3, S_{0}=1$ ). 


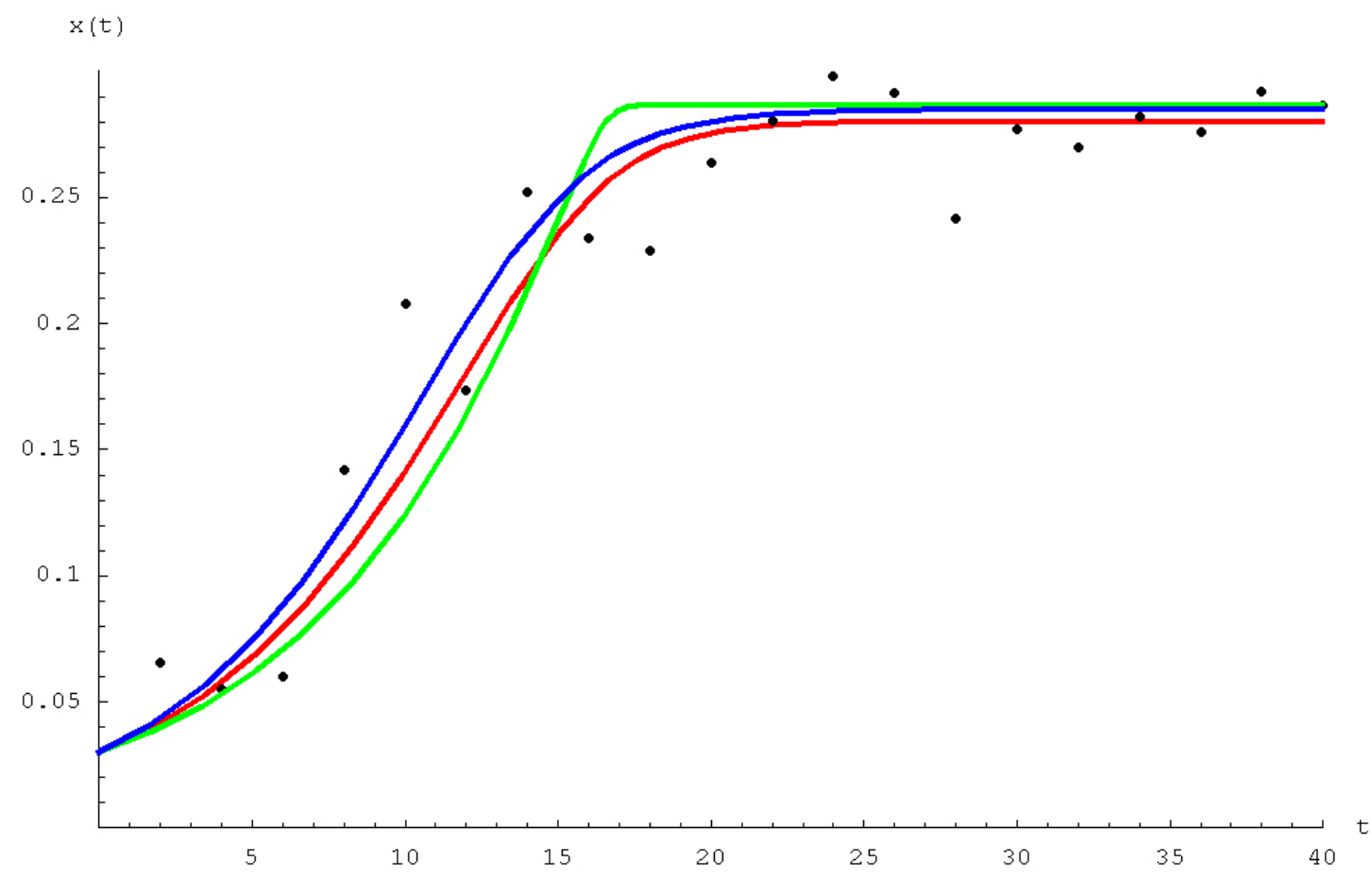

Figure 7: Illustration of the correlation of parameter estimations problem. Three solutions of the Monod model with different parameters but same least square value from the artificial experimental data set: red: $(0.25,0.5,0.25)$ true parameter set; green: $(0.1497,0.047,0.2565)$; blue: $(0.3418,0.8224,0.2547)$. 
2003; Holmberg, Ranta, 1982; Chappel, Gofrey, 1991). The minimum values of the least squares estimation for the regression model in a two dimensional problem give an ellipse, and if the estimates of the parameters are closely correlated (as $\widehat{\theta}_{1}$ and $\widehat{\theta}_{2}$ ) then the ellipse is very elongated (Figure 2,3); when parameters are not correlated this regression ellipse is a circle. If we consider a problem of 3 parameters then the resulting object is a 3 dimensional ellipsoid. But in this case parameter $\theta_{3}$ is estimated very well by any experimental design which includes measurements of the stationary phase of microbial growth. Then our problem is two dimensional, i.e. we need to estimate $\theta_{1}, \theta_{2}$. The eigenvalues of the Fisher information matrix are determined by the axes of this ellipse and their lengths are proportional to the square root of the inverse of the eigenvalues. The determinant of the Fisher information matrix is the area of this regression ellipse. Our objective is to find an optimal experimental design in order to make this regression ellipse small in some sense.

It was proved in (Dette et al., 2003) that the local D-optimal design for the Monod model is a 3 -point design. Therefore to identify the Monod parameters it is enough to take measurements of the growing biomass only 3 times in the optimal time. The two points of the optimal design are on the lower and upper bends and the third point is on the horizontal asymptote. We can give a nonformal interpretation of this optimal design as follows. To obtain good estimates of our parameters we should make measurements at points of the microbial growth curve where the curve changes its behavior determined by the parameters, i.e. the maximal specific growth rate determines the exponential phase of growth (Figure 4) and Michaelis-Menten constant determines the retardation phase of microbial growth (Figure 5). Then to obtain good estimates of those parameters it is necessary to take measurements at some critical points in the middle of those growth phases (i.e. in the middle of the lower and the upper bends). As for the Yield coefficient, then it is obvious that this parameter can be determined from one measurement of the stationary growth phase (Figure 6 ). It seems truly amazing that the results obtained with advanced mathematical techniques can be explained in such a simple and obvious way.

Now having constructed the optimal designs we are facing several very important questions, which will determine how useful the optimal experimental design approach is for the Monod model. Some of the questions are theoretical, some practical.

1) How efficient is the application of the non-linear optimal design in our experiments? Do we obtain a significant advantage from the application of those methods or may be some naive experimental designs (as the uniform design) be nearly as good?

2) What is the relation between local $D$-optimal design and other types of local optimal experimental designs? Maybe, for example, local E-optimal design will turn out to be more efficient in this case.

3) How precise should measurements be at optimal points? How stable is the optimal design with respect to experimental errors in the measurements? 
4) The initial value of parameters $\theta^{0}$ is crucial for the construction and performance of the optimal design, What will the result of the experiment if this value deviates substantially

from the 'true' value of the parameters? How will the optimal design change if the actual values of the parameters are not very close to the initial values?

Each of those questions is important in some sense. If this type of optimal design is not efficient compared to some naive experimental designs or if a much more efficient optimal design of another type exists we can reject this approach. If an optimal design is very efficient but not stable with respect to the experimental measurement error or is very sensitive to a change in the values of initial parameters, the design may also be inappropriate for practical purposes.

For optimal experimental design in the Monod model all those questions are answered in the affirmative and the answers are very positive. The improved efficiency of the obtained optimal design over the previous uniform design is obvious from the parameters estimation data (Table 1). The measurements in 3 optimal points provide much better approximations of the parameter $\theta^{*}$ than the measurements in 20 uniform time points! More statistically significant results relating the efficiency of the constricted optimal design can be found in the literature (Dette et al., 2003). The comparison of different optimal designs, also has been done (Dette et al., 2002). It has been found that E- and $e_{2}$ - optimal designs are more efficient than $D$-optimal designs for estimating the parameters $\theta_{1}$ and $\theta_{2}$ but the $D$-optimal design is more efficient for the estimation of $\theta_{3}$. However, if improvement of the accuracy in the estimation of the parameters $\theta_{1}$ and $\theta_{2}$ is considered to be more important, then the $E$ - and $e_{2}$-optimal designs have some advantages (Dette et al., 2002). The third and fourth questions also have been answered (Dette et al., 2002, 2003). It was found that optimal designs for the Monod model are robust with respect to the experimental errors and with respect to moderate misspecifications of the initial parameter.

Several theoretical considerations for microbiological models related to the Monod model have been published. Munack (1989) calculated the optimal feeding strategy for identification of Monod-type models using the modified E-criterion. Versyck, with colleagues (Versyck, 1998), considered the fed-batch reactor and compared modified E-criterion for Monod and Holdane kinetics. A theoretical comparison of those two models, including consideration of optimal experimental designs for parameter estimation and optimal control of the microbiological reactor, was undertaken by Smets with colleagues (Smets et al., 2002), where the modified E-criterion and D-criterion were under consideration. Berkholz, with colleagues (Berkholz et al., 2000), applied modified E-criterion in order to identify parameters of fed-batch hyaluronidase fermentation by Streptococus agalactiae. The application of optimal experimental design for parameter estimation of the Monod model was considered by Vanrolleghem with coauthors (Vanrolleghem et al., 1995) for activated sludge respiration. Growing microbial biomass in fed-batch experiments was characterized by their oxygen uptake with a respirograph biosensor. The single Monod model in specific form was successfully applied to the description of activated sludge kinetics:

$$
\frac{d S_{1}}{d t}=-\frac{\mu_{\max } X}{Y_{1}} \frac{S_{1}}{K_{m 1}+S_{1}}
$$




$$
O U R_{e x}=-\left(1-Y_{1}\right) \frac{d S_{1}}{d t}
$$

where $S_{1}$ denotes the substrate concentration, $X$ is the biomass concentration, $Y_{1}$ the yield coefficient, $\mu_{\max }$ the maximal specific growth rate, $K_{m 1}$ the saturation affinity constant and $O U R_{e x}$ the exogenous oxygen uptake rate. The important assumption in this model is that the biomass concentration $X$ is considered as a constant, because the ratio $S_{1} / X$ is low and biomass growth can be neglected $\left(\frac{d X}{d t}=0\right)$. The efficiency of the D- and E- optimal designs for the estimation and identification of the Monod parameters was demonstrated in the experiments of organic substrate (acetate was chosen) utilization by activated sludge.

D-optimal designs have been very useful for the identification of parameters of the nitrification model for activated sludge batch experiments (Ossenbruggen et al., 1996). The respiration rate of ammonium and nitrite was presented as a sum of two Michaelis-Menten functions, that is

$$
q_{0}=\frac{k_{N H} S_{N H}}{K_{N H}+S_{N H}}+\frac{k_{N O} S_{N O}}{K_{N O}+S_{N O}}
$$

where $q_{0}$ denotes the specific respiration rate (oxygen consumption), $S_{N H}$ is the ammonium $\left(\mathrm{NH}_{4}^{+}\right)$ concentration, $S_{N O}$ the nitrite $\left(\mathrm{NO}_{2}^{-}\right)$concentration, $k_{N H}, k_{N O}$ the maximum specific respiration rates, and $K_{N H}, K_{N O}$ the half saturation constants for $S_{N H}$ and $S_{N O}$, respectively. A D-optimality criterion was used to find good designs to model the two step nitrification process with piece-wise non-linear models of ammonium and nitrite concentration. The experimental part included a series of 14 batch runs where activated sludge was spiked with ammonium chloride and sodium nitrite individually and in combination with recording of respiration activity.

Another model for activated sludge process is the ASM Model No.1 incorporating carbon oxidation, nitrification and denitrification processes (Vanrolleghem et al., 1999; Hidalgo, Ayesa, 2001). This model describes the wastewater treatment process by activated sludge and is based on the Monodtype kinetics. The complete model contains 13 variables and 19 parameters. The structural identifiability and the optimal experimental design problem for the simplest modifications with two or three unknown parameters of ASM Model No.1 were investigated (Dochain et al., 1995; Vanrolleghem et al., 1999; Hidalgo, Ayesa, 2001).

Effective application of optimal experimental design to the anaerobic degradation of pollutants was developed by Merkel with colleagues (Merkel et al., 1996). The Monod type equation describes kinetics of anaerobic biodegradation of acetic acid in the batch and fed-batch experiments. The application of the optimal design technique allowed for accurate estimation of Monod parameters. The Monod or Michaelis-Menten type of non-linearity is incorporated in a large number of mathematical models used in biomedical sciences. The ideas of the optimal experimental design can be very useful for such situations. For example, the D-optimal design is efficient for the model based on the Michaelis-Menten equation with first-order decay of the enzyme describing batch enzymatic reaction where enzyme activity decades with time (Malcata, 1992). The problem of optimal design was considered many times from theoretical perspective and also experimentally for 
ligand-receptor interactions, enzymatic reactions and pharmacokinetics described by the models related to the Michaelis-Menten model (Johnson, Berthouex, 1975; Zaitcev et al., 1987; Rovati et al., 1988; Dunn, 1988; Dette, Wong, 1999; Hooker et al. 2003; Foracchia et al., 2003, Murphy et al., 2003).

\subsection{Application of optimal experimental design in microbiological mod- els}

Numerous mathematical models other then the Monod are used in microbiology to describe kinetic processes. Practically all the famous theoretical biology growth models (such as Logistic, Gompertz, Exponential models etc.) and the usual empirical regression models (linear, polynomial, exponential, different probability distributions etc.) have been applied to study processes in microbiology. Some of them were modified and their parameters have special names and precise meanings in particular areas of microbiology. There are many analytical considerations of the optimal experiment design problem for models which are used in microbiology (Table 2) and several very successful examples of application of this technique in experimental practice.

\subsubsection{Empirical regression models and growth models}

\section{Linear regression models}

Linear regression models are often used in microbiology and science in general. The optimal experimental design problem for the linear model is one of the classical and very extensively investigated problems in the experimental design area (Fedorov, 1972; Pukelsheim, 1993). The classical example of the successful application of D-optimal experimental design for the linear model in microbiology is the design of an experiment for DNA extraction from the anaerobic rumen microbial community (Broudiscou et al., 1998). The objective of the experiment is to assess the efficiency of the enzymatic pretreatment and to screen a number of detergents to maximize cell breakage and DNA recovery rate. Several potential detergents were tested: two enzymes (lysozyme and proteinase K) and five detergents (CHAPS, deoxycholic acid (SD), sodium dodecyl sulfate (SDS), sodiun lauroyl sarcosine (SLaS) and triton X-100). The following linear model with seven factors was applied to optimize DNA recovery

$$
\begin{aligned}
y & =b_{0}+b_{1} *(\text { Proteinase } \mathrm{K})+b_{2} *(\text { Lysozime })+b_{3} *(\mathrm{CHAPS})+b_{4} *(\mathrm{SD}) \\
& +b_{5} *(\mathrm{SDS})+b_{6} *(\text { Triton } \mathrm{X}-100)+b_{7} *(\mathrm{SLaS}) .
\end{aligned}
$$

Here $b_{i}, i=0, \ldots, 7$ are the unknown coefficients to be estimated. Also the variables 'Proteinase K' and 'Lysozime' were mutually excluded, as they relate to the same factor. The D-optimal design was numerically calculated by the NEMROD software (LPRAI, Universite Aix-Marseille III, France). This method gave the optimal quantity of detergents for maximization of DNA recovery. 


\section{Exponential models}

Exponential models are very often used for describing growth and death of microorganisms, doseresponse analysis and risk assessment (Coleman, Marks, 1998), and kinetics of metabolite production. Exponential models are also incorporated in the numerous model in predictive microbiology for describing effects of temperature (Geeraerd et al., 2000). The simplest single exponential model for microbial growth can be presented as a first-order differential equation

$$
\frac{d X}{d t}=\mu_{m} X
$$

where, $X$ is a biomass concentration and $\mu_{m}$ the maximal specific growth rate. In this context the closely associated parameter, called the doubling time $\tau$, is very often used in microbiology, i.e.

$$
\tau=\frac{\ln 2}{\mu_{m}} .
$$

The more complicated exponential models, including sums of several exponential functions, are also applied. For example, the model (Cobaleda et al., 1994), which is sum of two exponential terms with four unknown parameters, was used to fit the kinetic data of fusion between Newcastle disease virus and erythrocyte ghosts, i.e.

$$
F(t)=A_{1}\left[1-\exp \left(-k_{1} t\right)\right]+A_{2}\left[1-\exp \left(-k_{2} t\right)\right]
$$

where, $A_{1}, A_{2}, k_{1}, k_{2}$ are constant (unknown) parameters and $t$ represents time. The first exponential equation represents a fast reaction, which is the viral protein-dependent fusion process itself, and the second exponential equation represents a slow nonspecific dequenching reaction (Cobaleda et al.1994). Another model, which is the sum of two exponentials with three unknown parameters, was used to describe Escherichia coli inactivation by pulsed electric fields (Alvarez et al., 2003). The biological meaning of this model is that in one population of microorganisms two subpopulations exist, where the first population is sensitive with respect to the inactivating factor and the second population is resistant (Pruitt, Kamau, 1993), i.e.

$$
S(t)=p \exp \left(-k_{1} t\right)+(1-p) \exp \left(-k_{2} t\right)
$$

where $S(t)$ denotes the fraction of total survivors, $t$ is the treatment time, $p$ is the fraction of survivors in population 1 (sensitive population), $(1-p)$ is the fraction of survivors in population 2 (resistant population) and $k_{1}, k_{2}$ are the specific death rates of subpopulation 1 and 2 respectively. The optimal design of experiments for some of the exponential-type models were analyzed many times and their theoretical properties are well known (Melas, 1978; Mukhopadhyay, Haines, 1994; Dette, Neugebauer, 1997; Han, Chaloner, 2003; Dette, Melas and Pepelyshev, 2003b). However, because the number of concrete practical applications is very small, several examples of applications of optimal designs for exponential models in food microbiology will be considered in Section 3.2 .2 of this paper. 


\section{Logistic models}

The logistic type models is traditionally very often used in biology and in particular in microbiology for the description dynamic of microbial growth, infection processes and risk assessment (Peleg, 1997; Coleman, Marks, 1998; Ratkowsky, 2002; Pouillot, 2003). The simple continuous logistic model for microbial growth can be written as a first-order non-linear differential equation, that is

$$
\frac{d X}{d t}=\mu_{m} X(1-k X)
$$

where, $X$ is a biomass concentration, $\mu_{m}$ is the maximal specific growth rate, and $k$ is a logistic growth constant. The biological meaning of the logistic growth constant $k$ is the inverse of the maximal possible biomass concentration, i.e. $K=\frac{1}{k}$.

The logistic model is one of the basic models in ecology, where it well-know as the VerhilstPearl equation. This equation describes density-dependent growth of the biological population (Nicholson, 1954). The parameters of the logistic equation are the specific growth rate $\left(r=\mu_{m}\right)$ and maximal size of population $(K=1 / k)$ and have fundamental meanings in ecology as they are the basis for the concepts of $r$ - and $K$ - selections (May, 1981). It is interesting to note that the logistic equation can be described as the first two terms of Taylor's expansion of the growth equation. We will shortly present this classical consideration as developed by Lotka (1925).

Let us assume that the population (microbial culture) growth rate at any moment is a function of the population size (number or concentration of microorganisms), i.e.

$$
\frac{d X}{d t}=f(X)
$$

Usually the function $f(X)$ can be expanded in a convergent Taylor series, that is

$$
\frac{d X}{d t}=c_{0}+c_{1} X+c_{2} X^{2}+\ldots
$$

If $X=0$ then $c_{0}$ should be equal 0 as well, because it is necessary that there is at least one microorganism for the population to grow. Then if we will set $f(X)=c_{1} X$, the exponential model for the microbial growth is obtained where the coefficient $c_{1}$ is the maximal specific growth rate $\mu_{m}$. The important property of this equation is that the zero growth rate may be registered only at $X=0$ and a positive growth rate $\frac{d X}{d t}>0$ is observed at any population size.

If the next term of the Taylor series is also under consideration, i.e.

$$
f(X)=c_{1} X+c_{2} X^{2}
$$

then the logistic equation, with the coefficients $c_{1}=\mu_{m}$ and $c_{2}=-\mu_{m} k$, is obtained. The important property of the logistic equation is that it is the simplest equation, where $f(X)$ has two roots, one at $X=0$ and the second when the number of microorganisms $X$ reaches some positive saturation level. This property provides a typical sigmoid growth curve. 
The logistic equation can be integrated by separation of variables and the solution is

$$
X=\frac{1}{k\left(1-e^{\alpha-\mu_{m} t}\right)},
$$

where $\alpha$ is the constant of integration, which determines the position of the solution relative to the origin. The asymptotic value $\lim _{t \rightarrow \infty} X=1 / k$ is the saturation level of the microbial culture (population) which can not be exceeded because of the limitations of cultivation or of the natural environment.

Another important variant of the logistic equations is the discrete logistic equation, which is named in ecology as the difference logistic equation. In this case the variable $t$ is not instantaneous time, but discrete time, and $\triangle t$ notation

is used instead of $d t$ notation. A difference logistic equation can be presented in the form

$$
X_{n+1}=r X_{n}\left(1-X_{n}\right) \quad, 0<X<1,
$$

where $X_{n}$ and $X_{n+1}$ are the fractions (of the habitat maximum capacity, for example) of a population in the time step, or generation, $n$ and $n+1$; and $r$ is the constant characterizing the population in the particular environmental conditions. Several modifications of this discrete logistic equation which are using in the microbiology, were recently considered by Peleg (1997). Furthermore there exist several differences equations which an analogous to the logistic equation as density-dependent equations (May, 1981).

Local optimal experimental designs for the logistic model based on the D- and E-criteria and Bayesian designs have been extensively investigated from a theoretical prospective (Chaloner, Larntz, 1989; Ford et al.1992, Sebastiani, Settimi, 1997). We will briefly consider the Bayesian optimal design problem for the logistic model in Section 4.

\section{The Hill model}

The Hill equation (Bezeau, Endrenyi, 1986; Liu, Tzeng, 2000) is an empirical equation widely applied in biomedical sciences. This equation was proposed by A.V. Hill in 1910 to describe the binding of oxygen to hemoglobin (Bezeau, Endrenyi, 1986). Since then it has been widely applied in physiology, pharmacokinetic modeling and as one of the growth equations (Savageau, 1980). In microbiology this equation, for example, has been successfully applied to describe sporulation kinetics of Bacillus thuringiensis (Liu, Tzeng, 2000). In terms of the enzyme kinetics the Hill equation describes the relationship between substrate concentration $(c)$ and reaction velocity $(v)$ :

$$
v=\frac{V c^{N}}{\left(K^{N}+c^{N}\right)},
$$

where $V$ is the maximal velocity, $K$ is the substrate concentration required to yield half of the maximal velocity (analogue of the Michaelis-Menten coefficient), and $N$ is the Hill coefficient, which is the measure of deviation from hyperbolicity. 
It is obvious that when $N=1$ the Hill model gives the Michaelis-Menten model. At the same time the Hill equation is equivalent to the 3-parameter logistic function (Pregibon, 1983)

$$
y=A \frac{e^{\alpha+\beta x}}{1+e^{\alpha+\beta x}},
$$

where $A=V, \beta=N, \alpha=-N \ln K$, and $x=\ln c$. The D-optimal experimental design for the determination of the parameters $V, K$ and $N$ of the Hill equation or, what is the same, $A, \beta$ and $\alpha$ of the logistic function was investigated by M. Bezeau and L. Endrenyi (1986). 3-point optimal designs were constructed numerically and several important properties of the design were considered. In particular, the effect of the additive error of the observed value was investigated under the assumption that the error can be described by the power model, in which the variance of the error is proportional to the 'true' response raised to some power, i.e.

$$
\sigma_{i}^{2}=\sigma_{0}^{2} v_{i}^{2 \lambda}
$$

where $0 \leq \lambda \leq 1$, and the variance $\sigma_{0}^{2}$ is the constant of proportionality. When $\lambda=0$ it reduces to the case of homogeneous variance and when $\lambda=1$ it reduces to the case of constant relative (or constant percentage) errors. The effect on the design efficiency of the parameter $\lambda$ and initial parameter values was investigated. It was found that D-optimal designs for the Hill equation are robust with respect to moderate deviations from the assumed parameter values and efficient for different values of $\lambda$. The optimal designs were compared with the uniformly and logarithmically spaced designs. In general, the optimal design was always better than uniform design, but 4-, 5-, 6 -point logarithmically designs also performed very well. The optimal design for the case $\lambda=0.5$ generally outperformed both uniformly and logarithmically spaced designs.

\section{Generalized microbial growth models}

The model of microbial growth developed by J. Baranyi and colleagues (Baranyi et al., 1993) is very often used to describe microbial growth in food. This growth model has some advantages compared to the other famous models of microbial growth in food, because it provides a better approximation of the lag-phase of microbial culture growth (Baranyi, Roberts, 1995). The crucial step in this approach is the consideration of the potential growth and actual growth concept. It is well know that in most experiments the potential growth rate of the culture is higher then the actual growth rate if the time is close to inoculation. J. Baranyi et al. (1993) suggested the use of the ratio of the actual to the potential growth in a given environment to characterize the process of adjustment of the cells to the new environment. The pre-inoculation environment (the subculture) is denoted by $E_{1}$ and the actual (post-inoculation) environment by $E_{2}$.

When this difference is neglected $\left(E_{1}=E_{2}\right)$ then the microbial growth is described by the usual first-order autonomous differential equation of the form

$$
\frac{d x}{d t}=\mu(x) x
$$


where $x$ is the concentration of microorganisms and $\mu(x)$ is the specific growth rate. The change of $\mu(x)$ is determined by the model of microbial kinetics; it may be any of the Exponential, Monod, Gompertz or Logistic equations.

But in some cases the difference between $E_{1}$ and $E_{2}$ is large, or very important, and it cannot be neglected. For such cases an adjustment function $\alpha(t)$ from $E_{1}$ to $E_{2}$ is introduced. This function depends on $E_{1}$ and $E_{2}, 0 \leq \alpha(t) \leq 1$ for $t \geq 0, \alpha(t) \rightarrow 1$, and monotone increasingly as $t \rightarrow \infty$. Also it is assumed that $\mu(x)$ is independent of $E_{1}$ and the same as in the classical autonomous models. Then the differential equation which describes microbial growth is the non-autonomous differential equation

$$
\frac{d x}{d t}=\alpha(t) \mu(x) x .
$$

The class of the adjustment functions that were suggested have the form

$$
\alpha_{n}(t)=\frac{t^{n}}{\lambda^{n}+t^{n}},
$$

where $\lambda$ and $n$ are positive numbers (Baranyi et al., 1993). It is obvious that those functions are special cases of the Hill equation when $V=1$. The problem of the identification of parameters of this model was considered by Grijspeerdt and Vanrolleghem (1999). The structural identifiability was investigated using the Taylor series expansion. Practical identifiability and optimal design for this model were also considered. The local D-optimal design was constructed numerically and its efficiency and robustness were investigated using Monte Carlo simulations. The obtained results demonstrated the potential effectivity of the optimal experimental design approach.

\subsubsection{Optimal experimental design in microbial inactivation processes}

Predictive microbiology (the quantitative microbial ecology of food) has received much attention during the last few decades. This relatively young research area is devoted to the analysis of microbial behavior in food (McMeekin et al. 2002). The central concept of predictive microbiology is that the growth, survival and death responses of microbes in foods should be modeled with respect to the main controlling factors, such as temperature, $\mathrm{pH}$ and water activity (Baranyi, Robert, 1994; Roberts, 1995).

There is a difference between modelling approaches used in biotechnology and environmental microbiology and those used in predictive microbiology. The main differences are (Roberts, 1995)

(i) The bacterial concentration of concern to food microbiologists is lower than that for fermentation microbiologists, and it may be inappropriate to use fermentation models.

(ii) The aim is different: in predictive microbiology it is important to prevent growth of microorganisms rather than optimize it.

(iii) In food microbiology, as in environmental microbiology, the controlling factors influencing growth are generally rather heterogeneous and often poorly quantified. 
(iv) Many foods are nutritionally rich, so nutrient limitation of microbial growth does not occur.

The classification techniques of the mathematical models used in predictive microbiology have unique features. Simple classification is based on the microbial population behavior (McMeekin and Ross, 2002) described growth models, limit of growth (interface) models and inactivation models.

Evaluation of the death of microorganisms is one of the most typical problems in food microbiology. Usually the experimental aspects of the research include a large number of experiments, where the main affective factor and other factors vary. Therefore application of optimal experimental designs is a way to significantly reduce the cost of the experiments and to improve the quality of the obtained results. The typical problem is the analysis of the temperature inactivation of microorganisms; different variations of temperature effects are most often used for growth inhibition or killing microorganisms. The mathematical theory of this process has received much attention in recent decades.

The first-order kinetic model is the simplest and is often useful for describing inactivation of microorganisms in one factor experiments. For example, at a constant temperature

$$
\frac{d N}{d t}=-k(T) N
$$

where $N$ is the microbial population number (or density in a homogeneous model), and $k$ is the specific rate of thermal inactivation, which is a constant at a given temperature. It is obvious that for this type of experiment the very thoroughly investigated optimal designs for the exponential regression model can be efficiently applied.

The Arrhenius-type model (Bigelow, 1921) and its subsequent modifications (Bernaerts, 2000) are very often used for determining the inactivation constant $k$, i.e.

$$
k(T)=\frac{2.303}{D_{\text {ref }}} \exp \left[\frac{2.303}{z}\left(T-T_{\text {ref }}\right)\right],
$$

where $D_{\text {ref }}$ is the decimal reduction time for the reference temperature $T_{r e f}$, and $z$ is the number of degrees of temperature change required for a tenfold change $\mathrm{D}$ value, i.e. decimal reduction time for an arbitrary temperature.

It is very common in food science to use this model and so the problem of the estimation of the parameters $D$ and $z$ is very important. Optimal experimental designs for this model were developed numerically using the D-criterion (Cunha et al., 1997, Cunha, Oliveira, 2000) and the modified E-criterion (Versyck et al., 1999) . It was demonstrated theoretically that the optimal experimental designs are efficient and to illustrate the concept, a case study has been done using data from the literature (Cunha et al., 1997).

Another way to inactivate microorganisms by temperature is to keep products under suboptimal temperatures for microbial growth. Mathematical models used for describing those processes are not principally different from models of heat inactivation. Among the most popular models is 
the Ratkowsky model (Ratkowsky et al., 1982) and its modifications (Baranyi, Robert, 1994). Bernaerts and colleagues (Bernaerts et al., 2000) not only demonstrated theoretical efficiency of the E-optimal design for experiments in the Ratkowsky-type model but also conducted some practical experiments with Escherichia coli K12 to illustrate the application of the optimal design. The model for growth of microorganisms as a function of temperature, proposed by Baranyi and Roberts (1994), is given by

$$
\begin{aligned}
\frac{d n}{d t} & =\frac{\theta(t)}{\theta(t)+1} \mu_{\max }[T(t)]\left[1-\exp \left(n(t)-n_{\max }\right)\right] \\
\frac{d \theta(t)}{d t} & =\mu_{\max }[T(t)] \theta(t)
\end{aligned}
$$

with an additional equation called the Square Root model, i.e.

$$
\sqrt{\mu_{\max }[T(t)]}=b\left(T(t)-T_{\min }\right)
$$

where $n(t)$ is the natural logarithm of the cell density (for homogeneous case), $n_{\max }$ is the natural logarithm of the maximal population density $N_{\max }$, and $\theta(t)$ is the variable related to the physiologic state of the microbial cells. The initial value $\theta(0)$ determines the lag phase duration which is assumed to be related to the physiologic condition of the inoculum. The maximum specific growth rate $\mu_{\max }$, as a function of the suboptimal growth temperature $T(t)$, is modelled by the two parameter model of Ratkowsky et al. (1982), where $b$ is a regression coefficient and $T_{\min }$ is termed the theoretical minimum growth temperature.

Theoretical results enabled the authors (Bernaerts et al., 2000) to construct such optimal dynamic temperature inputs, where the parameters of the Ratkowsky square root model were identified from a single set of cell density measurements. In this particular case the model parameters are uncorrelated, which significantly simplifies the mathematical computations and efficiency of the optimal design technique. In a later paper they demonstrated (Bernaerts et al., 2002) that a theoretical design with the modified E-criterion of the optimal temperature input was very efficient and relevant to real experimental applications. Constrained optimal temperature input guaranteed model validity and yielded accurate Square Root model parameters. The experimental part of the research included growth experiments with E. coli K12 in a computer-controlled bioreactor under optimal-valued temperature conditions and compared well with the classical static experimental design.

The probabilistic approach for the analysis of the thermal inactivation considers lethal effects as probabilities (Turner, 1975; Peleg, Cole, 1998). This approach is alternative to the deterministic consideration of the microbial inactivation kinetics. The initial assumptions of the deterministic approach relate to the statements that the number of microorganisms is so large that it is possible to consider the process as deterministic where each cell has the same probability of dying. Under those assumptions, the application of the differential equations, such as, for example, the first order kinetics, is appropriate. In the probabilistic approach the death of each cell is considered as a 
single event and the "death curve" is not a solution of a deterministic equation, but a probabilistic distribution (Turner, 1975; Peleg, Cole, 1998; Martinus, van Boekel, 2002). At the same time, there is a general theory of growth models developed in theoretical biology, where a natural connection between the probabilistic and deterministic approaches and different types of growth models is established (Turner et al., 1976; Turner, Pruitt, 1977; Savageau, 1979; Chen, Christensen, 1985). In particular the Monod equation under certain assumptions can be transformed into a special case of the logistic probability distribution (Christensen, Nyholm, 1984).

The logical consideration of the probabilistic inactivation process leads to the Weibull distribution as the most appropriate mathematical model for the interpretation of survival curves (Christensen, 1984; Peleg, Cole, 1998). In general, the Weibull distribution is widely used for reliability data, particularly the strengths of materials and failure times (Smith, 1991). In biology this distribution has been used as one of the growth models (Savagean, 1980), for dose-response function in toxicological studies (Christensen, 1984), and for the analysis of the microbial survival curves after different deactivation processes (Peleg, Cole, 1998; Peleg, 2000; Martinus, van Boekel, 2002; Collado et al., 2003). Other types of probabilistic distributions are also often applied for survival process analyses (Peleg, Cole, 1998; Peleg, 2000), but the Weibull distribution has some natural advantages such as flexibility in fitting experimental data and using a minimal number of parameters (Martinus, van Boekel, 2002). The probability density function of the Weibull distribution (Smith, 1991) is given by

$$
f(t)=\left\{\begin{array}{c}
\frac{\beta}{\alpha}\left(\frac{t}{\alpha}\right)^{\beta-1} \exp \left(-\left(\frac{t}{\alpha}\right)^{\beta}\right), t>0 \\
0, t \leq 0
\end{array},\right.
$$

where $\alpha, \beta>0$. The D-optimal experimental design for estimation of the kinetics parameters of processes described by the Weibull probability distribution function was considered by Cunha and colleagues (Cunha et al., 1998). The optimal designs were numerically constructed for two different types of experiments. The first was the Weibull process of microbial thermal death under the constant temperature. The optimal design in this case consisted of the set of two time sampling points, where fractional concentrations are irrational numbers (approximately $\eta_{1}=0.7032$ and $\left.\eta_{2}=0.19245\right)$ such that their product equals $\frac{1}{e^{2}}$. The second experimental situation consisted of isothermal experiments conducted over a range of temperatures. In this case the rate parameter $\alpha$ of the Weibull distribution depended on temperature and followed the Arrhenius-type behavior

$$
\frac{1}{\alpha_{i}}=\frac{1}{\alpha_{0}} \exp \left(-\frac{E a}{R T_{i}}\right)
$$

At the same time, the parameter of the Weibull distribution $\beta$ indicates the kinetic pattern and is independent of the temperature, within a limited range of temperatures. Those assumptions were confirmed in numerous published experiments (Cunha et al., 1998). Therefore, the kinetic parameters for this practical situation are $\alpha_{0}, E a$ and $\beta$. In this case the optimal design required not only the selection of sampling times but also the selection of optimal temperatures. It was 
found that the most informative measurements will be obtained in two isothermal experiments at the limit temperatures of the range of interest. In one experiment, two samples should be taken with the same conversions as in the first discussed experiment with constant temperature $\left(\eta_{1}=0.7032\right.$ and $\left.\eta_{2}=0.19245\right)$, while in the other experiment one sample should be taken at the $\eta_{3}=\frac{1}{e}$. Several case studies, based on published data, of the application of optimal experimental design for the Weibull model were presented by Cunha et al. (1998).

\section{Bayesian methods for regression models}

The Bayesian methods are receiving much attention in biomedical sciences and pharmacokinetics (Merle, Mentre, 1997) and ecological research (Bois, 2001; Dowl, Meyer, 2003; Marin et al., 2003). Bayesian methods also play a role in applications to the different networks such as Markov chains describing bacteria dynamics in the drinking-water distribution systems (Bois et al., 1997) and estimation of parameters of the growth of Listeria monocytogenes in milk (Pouillot et al., 2003), neural probabilistic networks for analysis of the growth phases of Bacillus subtilis under the Gompertz model assumption (Simon, Karim, 2001) and spatial structure of plant populations (Marin et al., 2003). The application of Bayesian methods for the the construction of good experimental designs in nonlinear regression models has been actively investigated in recent decades; it was considered in connection with the Michaelis-Menten type model in enzyme kinetics (Murphy et al., 2002, 2003), with the exponential and related first-order equations (Chaloner, 1993; Mukhopadhyay, Haines, 1995; Dette and Neugebauer, 1997; Sivaganesan et al., 2003), and with the logistic equation (Chaloner, 1993; Dette, Neugebauer, 1996).

The Bayesian design approach is based on the idea that a prior distribution for the unknown parameters can be specified. The posterior distribution of $\theta$ given the observations at the experimental conditions is then used for point estimations, hypothesis testing and prediction.

To be more specific, let us consider the Bayesian approach for the example of the Logistic regression which is very well investigated (Chaloner, Larntz, 1989; Chaloner, 1993; Dette, Neugebauer, 1996; Sun et al. 1996; Smith, Ridout, 2003). The logistic regression model can be presented as a problem of estimating the probability of success in the Bernoulli trial from a set of observations depending on an explanatory variable $x$. The data is given in the set $Y=\left\{y_{1}, . ., y_{n}\right\}$. The two unknown parameters are $\mu, \beta$, i.e. the vector $\theta=\{\mu, \beta\}$, and the probability of success is given by

$$
p(x, \theta)=1 /(1+\exp (-\beta(x-\mu)) .
$$

The parameter $\mu$ is the value of $x$ when the probability of success is 0.5 . In microbiology and toxicology this parameter has a very important meaning, namely the $50 \%$ level of the effect and is denoted by LD50. The second parameter $\beta$ is the slope in the logit scale.

Under some realistic assumptions it can be shown that the posterior distribution of $\theta$ is approximately a multivariate normal distribution with mean equal to the maximum likelihood estimate 
of $\theta$, say $\widehat{\theta}$. Moroever, the covariance matrix is equal to the inverse of the observed Fisher information matrix, i.e. the matrix of the second derivatives of the log likelihood function evaluated at the maximum likelihood estimate of $\theta$. It is also important to note that, for logistic regression, the observed and expected Fisher information matrixes are identical. (Chaloner, Larntz, 1989). For an experimental design $\xi$ on $X$, with $n$ distinct design points $x_{i}, i=1, \ldots n$ and proportions $\omega_{i}$ such that $\sum_{i=1}^{n} \omega_{i}=1$, define (Chaloner, Larntz, 1989)

$$
\begin{aligned}
p_{i} & =p\left(x_{i}, \theta\right)\left(1-p\left(x_{i}, \theta\right)\right), \\
t & =\sum_{i=1}^{n} p_{i} \omega_{i}, \\
\bar{x} & =t^{-1} \sum_{i=1}^{n} p_{i} \omega_{i} x_{i}, \\
s & =\sum n_{i} \omega_{i}\left(x_{i}-\bar{x}\right)^{2},
\end{aligned}
$$

here $t, \bar{x}$, and $s$ all depend on the unknown parameter $\theta$. The normalized Fisher information matrix $I(\theta, \eta)$ is given by

$$
I(\theta, \eta)=\left(\begin{array}{cc}
\beta^{2} t & -\beta t(\bar{x}-\mu) \\
-\beta t(\bar{x}-\mu) & s+t(\bar{x}-\mu)^{2}
\end{array}\right)
$$

Because the matrix $I(\theta, \eta)$ is non-singular, the posterior distribution of $\theta$ using the design $\eta$ is approximately multivariate normal with mean $\widehat{\theta}$ and covariance matrix $\left.(n I(\widehat{\theta}, \eta))^{-1}\right)$. Therefore preposterior expected losses can be approximated using the prior distribution of $\theta$ as the predictive distribution of $\widehat{\theta}$.

Several different criteria can be used to determine an optimal experimental design [see Chaloner and Larntz (1989), Chaloner and Verdinelli (1995) or Dette and Neugebauer (1996, 1997)]. For example the Bayesian $D$-optimal is defined as the design, which maximizes the function

$$
\phi_{1}(\eta)=E_{\theta} \log \operatorname{det} I(\theta, \eta) .
$$

An alternative criterion is to maximize the function

$$
\phi_{2}(\eta)=-E_{\theta}\left(\operatorname{tr} B(\theta) I(\theta, \eta)^{-1}\right),
$$

where $B(\theta)$ is a given symmetric 2 by 2 matrix. In the particular case where only linear combinations of the parameters are of interest, the matrix $B(\theta)$ does not depend on $\theta$ and is a matrix with known entries. If non-linear combinations of the parameters $\theta_{i}$ are of interest then $B(\theta)$ has entries which are functions of $\theta$. Some Bayesian interpretation of these criteria in terms of minimization of expected loss can be found in Chaloner and Verdinelli (1995).

The theoretical efficiency of those criteria had been demonstrated by Chaloner and Larntz (1989). They considered the uniform and independent prior distributions of $\beta$ and $\eta$. The results of these 
authors suggested that the Bayesian methodology for this model is preferable to the classical optimal experimental design procedure based on the best guest of parameters values, such as for example the local D-optimal design.

The important particular case of the logistic model when the slope $\beta$ is assumed to be known was considered by Dette and Neugebauer (1996). They proved that under a rather general assumption concerning the prior distribution $p_{\xi}(\theta)$, the Bayesian D-optimal design for the logistic regression with one unknown parameter is the one point design at the point $x^{*}$ which is given by the unique solution of the equation

$$
\int_{\theta} \frac{2 e^{x-\theta}}{1+e^{x-\theta}} p_{\xi}(\theta) d \theta-1=0
$$

The numerical investigation of the same one parametric regression model have shown that Bayesian design of experiments is an efficient tool for the statistical inference in nonlinear models (Sun et al., 1996).

Unfortunately Bayesian methods are not very often applied in microbiological practice despite their potential effectiveness and well developed theoretical background. However several very interesting examples of practical applications have been published recently. Bois with colleagues (1997) applied a Bayesian approach to the network describing bacterial growth in the drinkingwater distribution system. Authors used a mechanistic compartmental model based on differential equations to describe bacterial populations behavior in the network, affected by dissolved organic carbon (DOC) and chlorine concentrations. The differential equations were based on the mass conversation laws for the chemical reaction involved. Free living biomass was increased by consumption biodegradable BOD, decreased by natural mortality and by killing by chlorine, also it was balanced that biomass can attach to the cement walls and come from shearing off the walls. Consumption of BOD were assumed to follow Monod's kinetics. A Bayesian approach was used to combine prior knowledge from the scientific literature about the possible range of values for each model parameter, and data from experiments, in the context of the dynamics model parameters. $A$ priory truncated lognormal or loguniform distributions were assigned to the parameters based on the literature. The joint posterior distribution of the parameters was determined numerically by application of the Markov chain Monte Carlo techniques. This method gave an approximate convergence in about 100000 iterations. Different realistic scenarios of the model system development were considered.

Pouillot with colleagues (2003) applied Bayesian methods for the analyses of growth of Listeria monocytogenes in milk under different temperatures of incubation. They developed a typical model for the predictive microbiology based on the logistic equation with time delay, which represented lag phase of microbial growth as a primary model and a secondary model which described the effect of incubation temperature on the maximal specific growth rate. Published growth data (total 124 growth curves) of Listeria monocytogenes in milk was used to estimate the prior distributions of parameters. Then using Markov chain Monte Carlo techniques with the WinBAGS software (MRC Biostatistics Unit) the posterior parameter distributions were obtained. 


\section{Conclusions}

The present paper presents some results for optimal experimental design for estimating parameters in nonlinear regression models, which are usually used in microbiology. Papers in the statistical literature devoted to this problem provide some justification for the application of the least squares method for estimating the unknown parameters in these nonlinear regression models. For a sufficiently large sample size the least squares estimates are consistent and its distribution can be approximated by a normal distrubution. Intensive simulation studies elucidated that for realistic sizes of experiments the theoretical covariance matrix of the least suares estimator is rather close to the sampling one. This observation provides a starting point for the construction and implementation of optimal experimental designs, which maximize or minimize an appropriate function of the theoretical covariance matrix. Considerable efforts were paid to construct and study local optimal designs. It is demonstrated that for many models in microbiology local optimal designs are not too sensitive with respect to the specification of an initial value for the unknown parameter. Moreover, the application of these designs yields a substantial improvement with respect to the accuracy of the estimates and a reduction or even to annihilation the correlation between them.

In many cases the number of different experimantal conditions defined by a local optimal design is equal to the number of parameters in the model. It is rather difficult and not always possible to prove this merely theoretically, but it was confirmed for most of the commonly used models in microbiology models by intensive empirical studies. Elaboration of more advanced Bayesian or maximin-optimal designs seems to be promising, because these designs are more robust with respect to a substantial misspecification of the unknown parameters by the experimenter. However, the determination of optimal designs with respect to these more sophisticated optimlity criteria is a substantially harder problem compared the local optimality criteria. So far Bayesian and maximin optimal designs were constructed only for the most simplest models with one or two nonlinear parameters.

It should be stressed that the implementation of optimal designs is a potentially very important opportunity to improve efficiency of experimental studies in microbiology without increasing the costs of the experiments. However, at present, there are only few real applications of optimal designs in microbiological practice. The authors hope that this paper will clearly demonstrate the practical benefits of the optimal design methodology for estimating parameters of nonlinear regression models in microbiology.

Acknowledgements. The work of H. Dette and V.B. Melas was supported by the Deutsche Forschungsgemeinschaft (SFB 475: Komplexitätsreduktion in multivariaten Datenstrukturen). The authors are also grateful to Prof. Charles Suffel (Stevens Institute of Technology), who read a preliminary version of this manuscript and made many extremely useful suggestions to improve 
the presentation. The authors would also like to thank S. Biedermann for some help with the graphical representations and Isolde Gottschlich, who carried out several corrections in a former version of the manuscript.

\section{References}

[1] Aborhey, S., Williamson, D. (1978). State and parameter estimation of microbial growth processes // Automatica, 14, 5, 493-498

[2] Alvarez, I., Virto, R., Raso J., Condon, S. (2003). Comparing predicting models for the Escherichia coli inactivation by pulsed electric fields // Innov. Food Sci. Emerg. Technol., $4,2,195-202$

[3] Atkinson, A.C., Cook, R.D. (1995). D-optimum designs for heteroscedastic linear models. // J. Amer. Statist. Assoc., 80, 204-212

[4] Baltes, M., Schneider, R., Sturm, C., Reuss, M. (1994). Optimal experimental design for parameter estimation in unstructured growth models // Biotechnol. Prog., 10, 5, 480-488

[5] Baranyi, J., Roberts, T. A. (1995). Mathematics of predictive food microbiology // Int. J. Food Microbiol., 26, 2, 199-218

[6] Baranyi, J., Roberts, T.A. (1994). A dynamic approach to predicting bacterial growth in food // Int. J. Food Microbiol., 23, 3-4, 277-294

[7] Baranyi, J., Roberts, T.A., McClure, P. (1993). A non-autonomous differential equation to model bacterial growth // Food Microbiol., 10, 1, 43-59

[8] Berkholz, R., Rohlig, D., Guthke, R. (2000). Data and knowledge based experimental design for fermentation process optimization // Enzyme Microb. Technol., 27, 10, 784-788

[9] Bernaerts, K., Servaes, R.D., Kooyman, S., Versyck, K.J., Van Impe, J.F. (2002). Optimal temperature input design for estimation of the Square Root model parameters: parameter accuracy and model validity restrictions // Int. J. Food Microbiol., 73, 145-157

[10] Bernaerts, K., Versyck, K.J., Van Impe, J.F. (2000). On the design of optimal dynamic experiments for parameter estimation of a Ratkowsky-type growth kinetics at suboptimal temperatures // Int. J. Food Microbiol., 54, 1-2, 27-38

[11] Bezeau, M., Endrenyi, L. (1986). Design of experiments for the precise estimation of doseresponse parameters: the Hill equation // J. Theor. Biol., 123, 4, 415-430 
[12] Bigelow, W.D. (1921). The logarithmic nature of thermal death time curves // J. Infect. Dis., 29, 528-536

[13] Biedermann, S., Dette, H., Pepelyshev, A. (2003). Maximin Optimal Designs for the Compartmental Model // Preprint Ruhr-Universität Bochum., http://www.ruhr-unibochum.de/mathematik3/preprint.htm

[14] Blok, J., Struys, J. (1996). Measurement and Validation of Kinetic Parameter Values for Prediction of Biodegradation Rates in Sewage Treatment // Ecotoxicol. and Environ. Saf., $33,3,217-227$

[15] Bois, F.Y. (2001). Applications of population approaches in toxicology // Toxicol. Lett., $120,1-3,385-394$

[16] Bois, F.Y., Fahmy, T., Block, J.-C., Gatel, D. (1997). Dynamic modeling of bacteria in a pilot drinking-water distribution system // Water Res., 31, 12, 3146-3156

[17] Broudiscou, L.-P., Geissler, H., Broudiscou, A. (1998). Estimation of the Growth Rate of Mixed Ruminal Bacteria from Short-term DNA Radiolabeling // Anaerobe, 4, 3, 145-152

[18] Chaloner, K. (1993). A note on optimal Bayesian design for nonlinear problems // J. Statist. Plann. Inference, 37, 2, 229-235

[19] Chaloner, K., Larntz, K. (1989). Optimal Bayesian design applied to logistic regression experiments // J. Statist. Plann. Inference, 21, 2, 191-208

[20] Chaloner, K. and Verdinelli, I. (1995). Bayesian experimental design: a review // Statist. Sci., 10, 273-304

[21] Chappell, M.J., Godfrey, K.R. (1992). Structural identifiability of the parameters of a nonlinear batch reactor model // Math. Biosci., 108, 2, 241-251

[22] Chen, Ch.-Y., Christensen, E.R. (1985). A unified theory for microbial growth under multiple nutrient limitation // Water Res., 19, 6, 791-798

[23] Chernoff H. (1953). Locally optimal design for estimator parameters. Ann. Math. Stat. , 24, 586-602

[24] Christensen, E.R. (1984). Dose-response functions in aquatic toxicity testing and the Weibull model // Water Res., 18, 2, 213-221

[25] Christensen, E.R., Nyholm, N. (1984). Ecotoxicological assays with algae: Weibull doseresponse curves // Envir. Sci. Technol., 18, 9, 713-718 
[26] Cobaleda, C., Garcha-Sastre, A., Villar, E. (1994). Fusion between Newcastle disease virus and erythrocyte ghosts using octadecyl Rhodamine B fluorescence assay produces dequenching curves that fit the sum of two exponentials // Biochem. J., 300, 2, 347-354

[27] Coleman, M., Marks, H.(1998). Topics in dose-response modeling // J. Food Prot., 61, 11, 1550-1559

[28] Collado, J., Fernandez, A., Rodrigo, M., Camats, J., Lopez, A.M. (2003). Kinetics of deactivation of Bacillus cereus spores // Food Microbiol., 20, 5, 545-548

[29] Cunha, L.M., Oliveira, F.A.R., Brandao, T.R.S., Oliveira, J.C. (1997). Optimal Experimental Design for Estimating the Kinetic Parameters of the Bigelow Model // J. Food Eng., 33, 1-2, 111-128

[30] Cunha, L.M., Oliveira, F.A.R., Oliveira, J.C. (1998). Optimal Experimental Design for Estimating the Kinetic Parameters of Processes Described by the Weibull Probability Distribution Function // J. Food Eng., 37, 2, 175-191

[31] Cunha, L.M. and Oliveira, F.A.R. (2000). Optimal experimental design for estimating the kinetic parameters of processes described by the first-order Arrhenius model under linearly increasing temperature profiles // J. Food Eng., 46, 1, 53-60

[32] Dette, H., Biedermann, S. (2003). Robust and efficient designs for the Michaelis-Menten model // J. Amer. Statist. Assoc., to appear.

[33] Dette, H., Haines, L., Imhof, L. (2003a). Maximin and Bayesian optimal designs for regression models // Preprint Ruhr-Universität Bochum, http://www.ruhr-unibochum.de/mathematik3/preprint.htm

[34] Dette, H., Haines, L., Imhof, L. (2003b). Maximin and Bayesian for heteroscedastic polynomial regression models // Preprint Ruhr-Universität Bochum, http://www.ruhr-unibochum.de/mathematik3/preprint.htm

[35] Dette, H., Melas, V.B., Pepelyshev, A., Strigul (2002). Efficient design of experiments in the Monod model. // Ruhr-Universität Bochum. http://www.ruhr-unibochum.de/mathematik3/preprint.htm. 30 pp.

[36] Dette, H., Melas, V.B., Pepelyshev, A., Strigul, N. (2003). Efficient design of experiments in the Monod model // J. R. Stat. Soc. B, 65, Part 3, 725-742

[37] Dette, H., Melas, V.B., Pepelyshev (2003a). Standardized maximin E-optimal designs for the Michaelis Menten model // Stat. Sinica, 13, 1147-1167 
[38] Dette, H., Melas, V.B., Pepelyshev (2003b). Locally E-optimal designs for exponential regression models // Preprint Ruhr-Universität Bochum., http://www.ruhr-unibochum.de/mathematik3/preprint.htm

[39] Dette, H., Melas, V.B., Wong, W.K. (2004). Optimal design for goodness-of-fit of MichaelisMenten enzyme kinetics // Preprint Ruhr-Universität Bochum., http://www.ruhr-unibochum.de/mathematik3/preprint.htm

[40] Dette, H., Neugebauer, H.-M. (1996). Bayesian optimal one point designs for one parameter nonlinear models // J. Statist. Plann. Inference, 52, 1, 17-31

[41] Dette, H., Neugebauer, H.-M. (1997). Bayesian D-optimal designs for exponential regression models // J. Statist. Plann. Inference, 60, 2, 331-349

[42] Dette, H., Wong, W.K. (1999). E-optimal designs for the Michaelis-Menten model // Statist. Probab. Lett., 44, 4, 405-408

[43] Dochain, D., Vanrolleghem, P.A., Van Daele, M. (1995). Structural identifiability of biokinetic models of activated sludge respiration // Water Res., 29, 11, 2571-2578

[44] Dowd, M., Meyer, R. (2003). A Bayesian approach to the ecosystem inverse problem // Ecol. Modell., 168, 1-2, 39-55

[45] Dunn, G. (1988). 'Optimal' designs for drug, neurotransmitter and hormone receptor assays // Stat. Med., 7, 7, 805-815

[46] Ellis, T.G., Barbeau, D.S., Smets, B.F., Grady Jr, C.P.L. (1996). Respirometric technique for determination of extant kinetic parameters describing biodegradation // Water Environ. Res., 68, 5, 917-926

[47] Fedorov, V.V. (1972). Theory of optimal experiments, Academic Press

[48] Ferenci, Th. (1999). "Growth of bacterial cultures" 50 years on: towards an uncertainty principle instead of constants in bacterial growth kinetics // Res. Microbiol., 150, 7, 431$-438$

[49] Foracchia, M., Hooker, A., Vicini, P., Ruggeri, A. (2003). POPED, a software for optimal experiment design in population kinetics // Comput. Methods Programs Biomed., In Press, Corrected Proof

[50] Ford, I., Torsney, B., Wu, C.-F.J. (1992). The use of a canonical form in the construction of locally optimal designs for nonlinear problems // J. R. Stat. Soc. B, 54, 2, 569-583

[51] Ford, I., Titterington, D.M., Wu, C.-F.J. (1985). Inference on sequential design // Biometrika, 72, 545-552 
[52] Ford, I., Silvey, S.D. (1980). A sequentially constructed design for estimating a nonlinear parametric function // Biometrika, 67, 381-388

[53] Fu, W., Mathews, A.P. (1999). Lactic acid production from lactose by Lactobacillus plantarum: kinetic model and effects of pH, substrate, and oxygen // Biochem. Eng. J., 3, 3, 163-170

[54] Geeraerd, A.H., Herremans, C.H., Van Impe, J.F. (2000). Structural model requirements to describe microbial inactivation during a mild heat treatment // Int. J. Food Microbiol., 59, 3, 185-209

[55] Goudar, C.T., Ellis, T.G. (2001). Explicit oxygen concentration expression for estimating extant biodegradation kinetics from respirometric experiments // Biotechnol. Bioeng., 75, $1,74-81$

[56] Grijspeerdt, K., Vanrolleghem, P. (1999). Estimating the parameters of the Baranyi model for bacterial growth // Food Microbiol., 16, 6, 593-605

[57] Guha, S., Jaffe, P.R. (1996). Determination of Monod Kinetic Coefficients for Volatile Hydrophobic Organic Compounds // Biotechnol. bioeng., 50, 6, 693-699

[58] Han, C., Chaloner, K. (2003). D- and c-optimal designs for exponential regression models used in viral dynamics and other applications // J. Statist. Plann. Inference, 115, 2, 585-601

[59] Hidalgo, M.E., Ayesa, E. (2001). A numerical identifiability test for state-space modelsapplication to optimal experimental design // Water Sci. Technol., 43, 7, 339-346

[60] Holmberg, A. (1982). On the practical identifiability of microbial growth models incorporating Michaelis-Menten type nonlinearities // Math. Biosci., 62, 1, 23-43

[61] Holmberg, A., Ranta, J. (1982). Procedures for parameter and state estimation of microbial growth process models // Automatica, 18, 2, 181-193

[62] Hooker, A.C., Foracchia, M., Dodds, M.G., Vicini, P. (2003). An evaluation of population D- optimal designs via pharmacokinetic simulations // Ann. Biomed. Eng., 31, 1, 98-111

[63] Hopkins, J.C., Leipold, R.J. (1996). On the dangers of adjusting the parameters values of mechanism-based mathematical models // J. Theor. Biol., 183, 4, 417-27

[64] Howell, J.A. (1983). Mathematical models in microbiology: mathematical tool-kit // In: Mathematics in microbiology, (1-37), Basin M. (eds.), London

[65] Jennrich, R.J. (1969). Asymptotic properties of non-linear least squares estimators. Ann. Math. Statist., 40, 633-643 
[66] Johnson, D.B., Berthouex, P.M. (1975). Efficient biokinetic experimental designs // Biotechnol. Bioeng., 17, 4, 557-570

[67] Karlin, S. Studden W. (1966). Tchebysheff Systems: with Application in Analysis and Statistics. New York, Wiley.

[68] Kiefer, J., Wolfowitz, J. (1960). The equivalence of two extremum problems. Canad. J. Math., 14, 363-366

[69] Leib, T.M., Pereira, C.J., Villadsen, J. (2001). Bioreactors: a chemical engineering perspective // Chem. Eng. Sci., 56, 19, 5485-5497

[70] Knightes, C.D., Peters, C.A. (2000). Statistical analysis of nonlinear parameter estimation for Monod biodegradation kinetics using bivariate data // Biotechnol. Bioeng., 69, 2, 160170

[71] Liu, B.L., Tzeng, Y.M. (2000). Characterization study of the sporulation kinetics of Bacillus thuringiensis // Biotechnol. Bioeng., 68, 1, 11-17

[72] Lotka, A.J. (1925). Elements of Physical Biology, Williams \& Wilkins

[73] Lopez-Fidalgo J. and W.K. Wong. (2002). Design Issues for the Michaelis-Menten Model // J. Theor. Biol., 215(1), 1-11

[74] Magbanua Jr, Benjamin S., Lu, Y.T., Leslie G. Jr, C.P. (1998). A technique for obtaining representative biokinetic parameter values from replicate sets of parameter estimates // Water Res., 32, 3, 849-855

[75] Malcata, F.X. (1992). Starting D-optimal designs for batch kinetics studies of enzymecatalyzed reactions in the presence of enzyme deactivation // Biometrics, 48, 3, 929-938

[76] Marin, J.M., Montes, D., Raquel and Rios Insua, D. (2003). Bayesian methods in plant conservation biology // Biol. Conserv., 113, 3, 379-387

[77] Martinus A.J.S., Van Boekel (2002). On the use of the Weibull model to describe thermal inactivation of microbial vegetative cells // Int. J. Food Microbiol., 74, 1-2, 139-159

[78] May, R.M. (1981). Theoretical ecology: Principles and Applications, Sinauer Associates

[79] McMeekin, T.A., Ross, T. (2002). Predictive microbiology: providing a knowledge-based framework for change management // Int. J. Food Microbiol., 78, 1-2, 133-153

[80] McMeekin, T.A., Olley, J., Ratkowsky, D.A., Ross, T. (2002). Predictive microbiology: towards the interface and beyond // Int. J. Food Microbiol., 73, 2-3, 395-407 
[81] Melas, V.B. (1978). Optimal designs for exponential regression // Mathematische Operationsforschung und Statistik, Series Statistics., 9, 1, 45-59

[82] Melas, V.B., Strigul, N.S. (1999). Investigation of the nonlinear regression Monod model by stochastic simulation / In: Statistical models with applications to econometrics and related fields, S.M. Ermakov and Yu.N. Kashtanov (eds.). Publishers of St. Petersburg State University, 118-127

[83] Merkel, W., Schwarz, A., Fritz, S., Reuss, M., Krauth, K. (1996). New strategies for estimating kinetic parameters in anaerobic wastewater treatment plants // Water Sci. Technol., 34, 5-6, 393-401

[84] Merle, Y., Mentre, F. (1997). Stochastic optimization algorithms of a Bayesian design criterion for Bayesian parameter estimation of nonlinear regression models: Application in pharmacokinetics // Math. Biosci., 144, 1, 45-70

[85] Monod, J. (1949). The growth of bacterial cultures // Ann. Rev. Microbiol., 3, 371-393

[86] Müller, Ch.H. (1995). Maximin efficient designs for estimating nonlinear aspects in linear models // J. Statist. Plann. Inference, 44, 117-132.

[87] Mukhopadhyay, S., Haines, L.M. (1995). Bayesian D-optimal designs for the exponential growth model // J. Statist. Plann. Inference, 44, 3, 385-397

[88] Munack, A. (1991). Optimization of sampling / In: Biotechnology, 4, 252-264, VCH Weinheim

[89] Murphy, E.F., Gilmour, S.G., Crabbe, M.J. (2003). Efficient and accurate experimental design for enzyme kinetics: Bayesian studies reveal a systematic approach // J. Biochem. Biophys. Methods, 55, 2, 155-178

[90] Murphy, E.F., Gilmour, S.G., Crabbe, M.J.C. (2002). Effective experimental design: enzyme kinetics in the bioinformatics era // Drug Discov. Today, 7, 20, 187-191

[91] Murphy, E.F., Gilmour, S.G., Crabbe, M.J.C. (2003). Efficient and accurate experimental design for enzyme kinetics: Bayesian studies reveal a systematic approach // J. Biochem. Biophys. Methods, 55, 2, 155-178

[92] Nicholson, A.J. (1954). An outline of the dynamics of animal populations // Austr. J. Zool., $2,9-65$

[93] Oh, S., Rheem, S., Sim, J., Kim, S., Baek, Y. (1995). Optimizing conditions for the growth of Lactobacillus casei YIT 9018 in tryptone-yeast extract-glucose medium by using response surface methodology // Appl. Environ. Microbiol., 61, 11, 3809-3814 
[94] Paulo M.J. and Rasch, Dieter A. M. K. (2002). Robustness and efficiency of D-optimal experimental designs in a growth problem. // Biom. J., 44, 5, 527-540

[95] Ossenbruggen, P.J., Spanjers, H., Klapwik, A. (1996). Assessment of a two-step nitrification model for activated sludge // Water Res., 30, 4, 939-953

[96] Peleg, M. (1997). Modeling microbial populations with the original and modified versions of the continuous and discrete logistic equations // CRC Crit. Rev. Food Sci. Nutr., 37, 5, $471-490$

[97] Peleg, M. (2000). Microbial survival curves - the reality of flat "shoulders" and absolute thermal death times // Food Res. Intern., 33, 7, 531-538

[98] Peleg, M., Cole, M. B. (1998). Reinterpretation of microbial survival curves // CRC Crit. Rev. Food Sci. Nutr., 38, 5, 353-380

[99] Petersen, B., Gernaey, K., Devisscher, M., Dochain, D., Vanrolleghem, P.A.(2003). A simplified method to assess structurally identifiable parameters in Monod-based activated sludge models // Water Res., 37, 12, 2893-2904

[100] Pirt, S.J. (1975). Principles of microbe and cell cultivation, Wiley, New York

[101] Poschet, F., Geeraerd, A.H., Scheerlinck, N., Nicolai, B.M., Van Impe, J.F. (2003). Monte Carlo analysis as a tool to incorporate variation on experimental data in predictive microbiology // Food Microbiol., 20, 3, 285-295

[102] Pouillot, R., Albert, I., Cornu, M., Denis, J.B. (2003). Estimation of uncertainty and variability in bacterial growth using Bayesian inference. Application to Listeria monocytogenes // Int. J. of Food Microbiol., 81, 2, 87-104

[103] Pregibon, D. (1982). Resistant fits for some commonly used logistic models with medical application // Biometrics, 38, 2, 485-498

[104] Pruitt, K., Kamau, D.N. (1993). Mathematical models of bacteria growth, inhibition and death under combinated stress conditions // J. Ind. Microbiol., 12, 221-231

[105] Pukelsheim, F. (1993). Optimal design of experiments, John Wiley \& Sons Inc.

[106] Pukelsheim, F., Torsney, B. (1991). Optimal designs for experimental designs on linearly independent support points // Ann. Statist., 19, 1614-1625

[107] Ratkowsky, D.A. (2002). Some examples of, and some problems with, the use of nonlinear logistic regression in predictive food microbiology // Int. J. Food Microbiol., 73, 2-3, 119-125 
[108] Ratkowsky, D.A., Olley, J., McMeekin, T.A., Ball, A. (1982). Relationship between temperature and growth rate of bacterial cultures // J. Bacteriol., 149, 1, 1-5

[109] Roberts, T.A. (1995). Microbial Growth and Survival: Developments in Predictive Modelling // Int. Biodeterior. Biodegradation, 36, 3-4, 297-309

[110] Rovati, G.E., Rodbard, D., Munson, P.J. (1988). DESIGN: computerized optimization of experimental design for estimating $\mathrm{Kd}$ and Bmax in ligand binding experiments. I. Homologous and heterologous binding to one or two classes of sites // Anal. Biochem., 174, 2, 636-649

[111] Saez, P.B., Rittmann, B.E. (1992). Model-parameter estimation using least squares // Water Res., 26, 6, 789-796

[112] Savageau, M.A. (1980). Growth equations: A general equation and a survey of special cases // Math. Biosci., 48, 3-4, 267-278

[113] Schirmer, M., Butler, B.J., Roy, J.W., Frind, E.O., Barker, J.F. (1999). A relative-leastsquares technique to determine unique Monod kinetic parameters of BTEX compounds using batch experiments // J. Contam. Hydrol., 37, 1-2, 69-86

[114] Sebastiani, P., Settimi, R. (1997). A note on D-optimal designs for a logistic regression model // J. of Statist. Plann. Inference, 59, 2, 359-368

[115] Silvey, S.D. (1980). Optimal design. Chapman and Hall, London.

[116] Simon, L., Nazmul K.M. (2001). Probabilistic neural networks using Bayesian decision strategies and a modified Gompertz model for growth phase classification in the batch culture of Bacillus subtilis // Biochem. Eng. J., 7, 1, 41-48

[117] Sivaganesan, M., Rice, E.W., Marinas, B.J. (2003). A Bayesian method of estimating kinetic parameters for the inactivation of Cryptosporidium parvum oocysts with chlorine dioxide and ozone // Water Res., 37, 18, 4533-4543

[118] Smets, I.Y.M., Versyck, K.J.E., Van Impe, J.F.M. (2002). Optimal control theory: A generic tool for identification and control of (bio-)chemical reactors // Ann. Rev. Control, 26, 1, 5773

[119] Smith, D.M., Ridout, M.S. (2003). Optimal designs for criteria involving log(potency) in comparative binary bioassays // J. of Statist. Plann. Inference, 113, 2, 617- -632

[120] Smith, R.L. (1991). Weibull regression models for reliability data // Reliability Eng. System Safety, 34, 1, 55-76 
[121] Sommer, H.M., Holst, H., Spliid, H., Arvin, E. (1995). Nonlinear parameter estimation in microbiological degradation systems and statistic test for common estimation // Environ. Int., 21, 5, 551-556

[122] Sun, D., Tsutakawa, R.K., Lu, W.-S. (1996). Bayesian design of experiment for quantal responses: What is promised versus what is delivered // J. of Statist. Plann. Inference, 52, 3, 289-306

[123] Turner, Jr., Malcolm E. (1975). Some classes of hit-theory models // Math. Biosci., 23, 3-4, 219-235

[124] Turner, Jr., Malcolm E., Bradley, Jr., Edwin L., Kirk, K,A., Pruitt, K.M. (1976). A theory of growth // Math. Biosci., 29, 3-4, 367-373

[125] Turner, Jr., Malcolm E., Pruitt, K.M. (1978). A common basis for survival, growth, and autocatalysis // Math. Biosci., 39, 1-2, 113-123

[126] Vanrolleghem, P.A., Spanjers, H., Petersen, B., Ginestet, Ph., Takacs, I. (1999). Estimating (combinations of) activated sludge model no. 1 parameters and components by respirometry // Water Sci. Technol., 39, 1, 195-214

[127] Vanrolleghem, P.A., Van Daele, M., Dochain, D. (1995). Practical identifiability of a biokinetic model of activated sludge respiration // Water Res., 29, 11, 2561-2570

[128] Versyck, K.J., Bernaerts, K., Geeraerd, A.H., Van Impe, J.F. (1999). Introducing optimal experimental design in predictive modeling: A motivating example // Int. J. Food Microbiol., $51,1,39-51$

[129] Versyck, K.J., Claes, J.E., Van I., J.F. (1998). Optimal experimental design for practical identification of unstructured growth models // Math. Comput. Simulation, 46, 5-6, 621-629

[130] Woodroofe, M., Coad, D.S. (2002). Corrected confidence sets for sequentially designed experiments: Examples // Sequential Analysis, 21, 191-218

[131] Wu, C.F.J. (1985). Efficient sequential designs with binary data. // J. Amer. Stat. Assoc. , 80, 974-984

[132] Zaitcev, S.V., Kurochkin, I.N., Varfolomeev, S.D. (1987). Discrimination of models and parameter estimation of the ligand-receptor interactions // In: Modern problems of biokinetics, 198-255, Moscow State University

[133] Zhang, T., Guay, M. (2002). Adaptive Parameter Estimation for Microbial Growth Kinetics // AIChE J., 48, 3, 607-616 